\title{
Dynamic Pricing and Inventory Management with Dual Suppliers of Different Lead Times and Disruption Risks
}

\author{
Xiting Gong \\ Department of Systems Engineering and Engineering Management, The Chinese University of Hong Kong, Shatin, N.T., Hong Kong \\ xtgong@se.cuhk.edu.hk \\ Xiuli Chao \\ Department of Industrial and Operations Engineering, University of Michigan, Ann Arbor, Michigan 48109, USA, xchao@umich.edu
}

Shaohui Zheng

School of Business and Management, Hong Kong University of Science and Technology, Clearwater Bay, Kowloon, Hong Kong imzheng@ust.hk

\begin{abstract}
$\mathrm{T}_{\mathrm{t}}^{\mathrm{t}}$ is common for a firm to make use of multiple suppliers of different delivery lead times, reliabilities, and costs. In this study, we are concerned with the joint pricing and inventory control problem for such a firm that has a quick-response supplier and a regular supplier that both suffer random disruptions, and faces price-sensitive random demands. We aim at characterizing the optimal ordering and pricing policies in each period over a planning horizon, and analyzing the impacts of supply source diversification. We show that, when both suppliers are unreliable, the optimal inventory policy in each period is a reorder point policy and the optimal price is decreasing in the starting inventory level in that period. In addition, we show that having supply source diversification or higher supplier reliability increases the firm's optimal profit and lowers the optimal selling price. We also demonstrate that, with the selling price as a decision, a supplier may receive even more orders from the firm after an additional supplier is introduced. For the special case where the quickresponse supplier is perfectly reliable, we further show that the optimal inventory policy is of a base-stock type and the optimal pricing policy is a list-price policy with markdowns.
\end{abstract}

Key words: inventory control; source diversification; optimal pricing; supplier disruption; list-price with markdown policy History: Received: December 2011; Accepted: January 2014 by Haresh Gurnani, Saibal Ray, and Yunzeng Wang, after 3 revisions.

\section{Introduction}

Supply disruption, which may result from various causes ranging from accidents and disasters, labor strikes, to quality issues and machine breakdowns, has been a challenge faced by many firms. In practice, source diversification and demand/pricing management have been two widely used strategies for mitigating the impacts of supply disruption. In this study, we are concerned with jointly determining pricing and inventory replenishment strategies for a firm that has dual unreliable supply sources and faces pricesensitive random demands. Due to possible disruption, the suppliers may or may not be able to deliver an order in a period, and the delivery capability of each supplier evolves in a Markovian nature over time. Motivated by the observation that many firms use a quick-response, but more costly, supplier that can deliver the firm's order relatively quickly, plus a less responsive and also less costly supplier for its regular supply (see, e.g., Fisher et al. 1994), in this study, we focus on the case where the delivery lead times for the two suppliers are zero (for the quickresponse supplier) and one (for the regular supplier) period of time, respectively, when they are capable of delivery. This also captures a popular setting where a firm has two supply sources: one is local or in-house production which tends to be more expensive, more reliable, and can deliver in a shorter time; while the other is non-local/outsourcing which tends to be less reliable and takes more time for delivery. We aim at (a) characterizing the optimal joint ordering and pricing policies that maximize the firm's expected total discounted profit over a planning horizon, and (b) analyzing the impacts of supply source diversification and supplier reliability on the firm's optimal profit, its optimal pricing and inventory policies, its customers, and its suppliers. 
This study is related to three streams of research literature: (i) procurement from multiple unreliable suppliers; (ii) inventory control with multiple delivery modes; and (iii) coordination of pricing and inventory decisions. Many researchers, including Anupindi and Akella (1993), Chen et al. (2001), Tomlin and Wang (2005), Dada et al. (2007), and Federgruen and Yang (2011), have studied inventory systems with multiple suppliers and random yield. The studies that are closely related to ours are those of dualsourcing inventory replenishment models, assuming that different suppliers may suffer different random disruptions and charge different unit costs. See, for example, Tomlin and Snyder (2007), Yang et al. (2009), Gürler and Parlar (1997), Chopra et al. (2007), Tomlin (2009), and Gumus et al. (2012). In particular, for the case where a firm has one perfectly reliable supplier and one unreliable supplier, Yang et al. (2009) discuss the value of information on the uncertain supplier's reliability; for the case when both suppliers are subject to disruptions, Gürler and Parlar (1997) derive an expression for the average cost objective function under a given $(q, r)$ policy, and then develop numerical approaches to evaluate the objective function for determining the optimal $q$ and $r$. All these studies assume zero lead time for all suppliers.

The second stream of related research is on inventory control with multiple delivery modes/supply lead times. In these studies, the research question addressed is the structure of optimal inventory policy for each supplier. Daniel (1963) is the first to consider a multi-period single-stage model with two shipping modes, with lead times being 0 and 1, respectively. Fukuda (1964) extends Daniel (1963) to the case where the lead times of the two supply modes are $L$ and $L+1$, respectively, for a general non-negative integer $L$. Whittemore and Saunders (1977) consider the dualsupplier problem with lead times of arbitrary lengths, and demonstrate that the optimal control policy is extremely complicated and the parameters of the optimal control policy are state-dependent if the difference in lead times is greater than 1. Feng et al. (2006) study the problem with multiple consecutive delivery modes, and show that only the fastest two modes have optimal base stock. Due to the complexity of the optimal policies with general lead times, among others, Veeraraghavan and Scheller-Wolf (2008) focus on the evaluation of two simple classes of heuristic policies, viz., "single index" and "dual index" policies. In all these studies, suppliers are assumed to be perfectly reliable and the selling price is assumed to be exogenously given.

The third stream of research related to our work is the coordination of pricing and inventory decisions. Several excellent survey studies in this area have been published (e.g., Chen and Simchi-Levi 2012); and the reader is referred to those sources for more discussions. In this line of research, the supplier is generally assumed to be reliable. See, for instance, Federgruen and Heching (1999), Zhao and Wang (2002), and Chen and Simchi-Levi (2004). There are only a few studies that consider joint pricing and inventory management for systems with supply uncertainty, including Li and Zheng (2006), Chao et al. (2008), Feng (2010), Feng and Shi (2012), Li et al. (2013a, 2013b), and Chen et al. (2013). Among them, the most related work to ours is Chen et al. (2013), in which the authors consider the joint optimal pricing and inventory control problem for a system with price-sensitive demands and multiple random-yield suppliers. Its major result is that the optimal inventory replenishment policy for each supplier is a near reorder point policy, that is, there exists a reorder point, above which no order is placed and below which a positive amount is ordered for almost every inventory level, and the optimal price may not be monotone in the starting inventory level.

The model in this study captures the several main features addressed in the above three streams of research (i.e., multiple sourcing, different lead times / shipping modes, and coordination of pricing and inventory decisions). The research issues addressed in this study include, besides the structure of the dynamic optimal pricing and inventory control policies, the impacts of supply source diversification and supplier reliability on the firm's optimal profit and operational policies, its customers, and its suppliers. We show that, when both suppliers are unreliable, the optimal inventory policy from each supplier is a reorder point policy and the optimal selling price in each period is decreasing in the starting inventory level of that period. In addition, we show that, diversifying supply source and/or improving supplier reliability increase the firm's expected profit and lower the optimal selling price; hence, they benefit both the firm and its customers. We also demonstrate that, with the selling price as a decision, a supplier may receive even more orders from the firm after an additional supplier is introduced, which seems counterintuitive and is different from the result in the case when the selling price is exogenously given. For the special case where the quick-response supplier is perfectly reliable, we further show that the optimal inventory policy is of a base-stock type for each supplier and the optimal pricing policy is a list-price policy with markdowns.

Although random disruption can be treated as a special case of random yield, compared to Chen et al. (2013), our model captures more features, such as Markovian uncertainties and different lead times of suppliers, and we obtain richer structures of the optimal policy. Moreover, in addition to deriving the optimal policy, we are able to identify the impacts of 
source diversification and suppliers reliabilities, which have not been addressed in Chen et al. (2013) or other related literature.

The contributions of this study are threefold. Firstly, this study is the first to consider the joint pricing and inventory control problem for a firm with dual unreliable suppliers of different lead times and Markovian disruption risks. We characterize the firm's optimal joint pricing and inventory policies and carry out several important sensitivity analyses. Second, this study is also the first to analyze the impact of source diversification on the firm, on its suppliers, and on its customers in a dynamic and stochastic setting. Our results align with economic intuitions that a more diversified supply benefits both the firm and its customers; however and interestingly, we also find that the incumbent supplier may receive more orders after a new supplier is introduced if the selling pricing is an endogenous decision. Lastly, this study is also the first to investigate the impact of supply reliability. Our results show that, in the presence of disruption risks, both the firm and its customers benefit from higher supply reliability, but this may not be true for the suppliers. Altogether, this study sheds light on how a firm should coordinate inventory, sourcing, and the selling price to manage disruption risks.

The remainder of this study is organized as follows. In section 2, we describe the model and introduce the dynamic programming formulation. In section 3, we characterize the structure of the optimal inventory and pricing policies and carry out several sensitivity analyses, which lead to some important insights on the impacts of dual sourcing and supply reliability. In section 4 we focus on the special case where the quick-response supplier is perfectly reliable, for which we have more structural results and additional insights. We conclude the study in section 5 with some discussions on possible extensions and future research. Finally, technical proofs and some numerical results are provided in the Appendix and the online Appendix S1.

\section{The Model}

A firm sells a single product over a planning horizon of $T$ periods, indexed by $t=1, \ldots, T$. The firm has two suppliers, referred to as supplier $k, k=1$, 2. Both suppliers face disruption risks in each period. When a supplier is not disrupted in a period, we say it is reliable, and in this case any order placed to this supplier will be fully delivered; if a supplier encounters a disruption in a period, however, then it will not be able to process the order and nothing will be delivered. Supplier 1 is a local/quick-response supplier; it can deliver any order in its entirety in the same period the order is placed (i.e., the lead time is zero) if there is no disruption. Supplier 2, on the other hand, is a nonlocal/regular supplier; it can deliver any order in its entirety in the next period (i.e., the lead time is one) if there is no disruption when it receives the order. This lead time structure is for analytical tractability. Suppose that the firm pays its suppliers for the delivered product only, and the unit ordering cost from supplier $k$ is $c_{k}$. Most of our results continue to hold when the firm pays its suppliers partially for the ordered product and partially for the delivered product. See section 5 for more discussions on this.

We model the random disruption process of supplier $k$ by a dependent Bernoulli process as follows: let $J_{t, k}$ denote supplier $k^{\prime}$ s delivery capability in period $t$, with $J_{t, k}=0$ indicating that supplier $k$ encounters a disruption in period $t$ and hence is unable to process any order in period $t$, and $J_{t, k}=1$ indicating that it is capable of processing an order in period $t$. We assume that $\left\{J_{t, k}, 0 \leq t \leq T\right\}$ forms a Markov chain with transition probabilities

$$
\begin{aligned}
P\left\{J_{t, k}=1 \mid J_{t-1, k}=l\right\} & =1-P\left\{J_{t, k}=0 \mid J_{t-1, k}=l\right\} \\
: & =\gamma^{k, l}, \quad l=0,1,
\end{aligned}
$$

where ":=" represents "defined as"; the two processes are independent of each other, which is due to the lead time/geographical difference between the two suppliers.

\section{REMARK 1.}

(a) Note that here $J_{t, k}$ represents the internal disruption status of supplier $k$, not the order delivery process. The order in period $t$ for supplier $k$ is processed and shipped if supplier $k$ is not disrupted in period $t$, and as long as the order is processed and shipped, it will be delivered to the firm in period $t$ for supplier 1 and in period $t+1$ for supplier 2 .

(b) The disruption process $\left(J_{t, 1}, J_{t, 2}\right)$ during period $t$ is random and is not known to the firm when it places order at the beginning of period $t$. However, after $\left(J_{t, 1}, J_{t, 2}\right)$ is realized during period $t$, we assume that it becomes public information and the firm would know $\left(J_{t, 1}, J_{t, 2}\right)$ at the beginning of period $t+1$ even if it did not place any order in period $t$. When this assumption is violated, then the firm would need to make efforts to acquire this information. Note that this is different from the case when a firm's reliability is observed by the firm upon delivery. The reader is referred to Parlar et al. (1995) for more discussions on this.

For notational convenience, it is assumed here the transition probabilities are time-independent, but all 
results hold when they depend on time. We further assume $\gamma^{k, 0} \leq \gamma^{k, 1}$ for $k=1$, 2, which implies

$$
\left\{J_{t+s, k} \mid J_{t-1, k}=0\right\} \leq_{s t}\left\{J_{t+s, k} \mid J_{t-1, k}=1\right\}, \quad \text { for } s \geq 0 \text {. }
$$

That is, $\left\{J_{t+s, k} \mid J_{t-1, k}=l\right\}$ is stochastically increasing in $l$. For more discussions on stochastic ordering, see Shaked and Shanthikumar (1994). Condition (1) simply means that supplier $k$ will be more likely to be reliable in the current and future periods if it was reliable in the previous period than if it was disrupted in the previous period.

The demand $D_{t}$ in period $t$ is a non-negative random variable and it depends on the selling price $p$ in the period. We assume an additive demand model with

$$
D_{t}=d_{t}(p)+\epsilon_{t}, t=1, \ldots, T
$$

where $\epsilon_{1}, \ldots, \epsilon_{T}$ are independent random variables with mean zero, and are also independent of both suppliers' delivery capabilities. Thus, the suppliers' delivery capabilities only impact demands through the selling prices. The mean demand $d_{t}(p)$ is positive and strictly decreasing in $p \in\left[\underline{p}_{t}, \bar{p}_{t}\right]$, where $\underline{p}_{t}$ and $\bar{p}_{t}$ are the lowest and highest possible unit prices to be charged, respectively. This additive demand model has been extensively discussed in the literature on joint optimization of pricing and inventory replenishment (see, e.g., Chen and Simchi-Levi 2012, Federgruen and Heching 1999, Feng 2010). Let $p_{t}(d)$ be the inverse function of $d_{t}(p)$ over $d \in\left[\underline{d}_{t}, \bar{d}_{t}\right]$, where $\bar{d}_{t}=d_{t}\left(p_{t}\right) \geq \underline{d}_{t}=d_{t}\left(\bar{p}_{t}\right)$. Thus, charging a selling price $p$ in period $t$ is equivalent to choosing the mean demand $d=d_{t}(p)$. In section 5 , we will provide some discussions for the case when the demand model has a more general additive-multiplicative form.

At the end of each period, any unsold product is carried to the next period with a holding cost; and any unsatisfied demand is backlogged with a backlog cost. Similar to Federgruen and Heching (1999), Chen and Simchi-Levi (2004), and Li and Zheng (2006), we assume that a customer pays the firm in the period the demand occurs, even if the demand is backlogged; any delay in delivery will be compensated with the backlog cost. Under these assumptions, the expected sales revenue of the firm in period $t$ is $R_{t}(d):=p_{t}(d) d$ when the mean demand $d$ is chosen. We assume $R_{t}(d)$ is a concave function of $d$ over $\left[\underline{d}_{t}, \bar{d}_{t}\right]$, which is a standard assumption in the literature (see, e.g., Federgruen and Heching 1999, Li and Zheng 2006).

The sequence of events in each period $t$ is as follows: First, delivery from supplier 2 (if any, and that supplier 2 was not disrupted in period $t-1$ ) arrives.
Second, the starting inventory level and two suppliers' delivery capabilities in period $t-1$ are reviewed. Third, the firm decides the order quantity for each supplier $k$, denoted as $q_{k}, k=1,2$, and sets the selling price $p$ (or equivalently, the mean demand $d$ ). Fourth, random delivery capabilities of the two suppliers in period $t, J_{t, 1}$, and $J_{t, 2}$, are realized. Fifth, depending on the realization of $J_{t, 1}$ delivery from supplier 1 arrives (if any) and the customer demand is realized. Finally, the sales revenue and all costs for the period are calculated. Suppose there is a one-period discount factor $\alpha \in(0,1]$. The firm's objective is to find the optimal joint pricing and inventory policies to maximize its expected total discounted profit over the planning horizon.

Let $G_{t}(z)$ denote the expected holding and backlog cost in period $t$ when the inventory level after satisfying the mean demand is $z$. We assume that $G_{t}(z)$ is convex in $z$. Clearly, the convexity is satisfied if the holding and backlog costs are linear in the ending inventory, that is,

$$
G_{t}(z)=h \mathbf{E}\left[\max \left\{z-\epsilon_{t}, 0\right\}\right]+b \mathbf{E}\left[\max \left\{\epsilon_{t}-z, 0\right\}\right],
$$

where $h$ is the unit holding cost, and $b$ is the unit backlog cost.

We define $(x, i, j)$ as the "state" of the system at the beginning of the period, where $x$ is the starting inventory level after receiving delivery from supplier 2 (if any), and $i$ and $j$ are suppliers 1 and 2's delivery capabilities in the previous period, respectively. Let $V_{t}(x, i, j)$ denote the firm's maximum expected total discounted profit-to-go from period $t$, also known as value function or optimal profit function, given the system state is $(x, i, j)$ at the beginning of period $t$. Then, from standard stochastic dynamic programming, we have, for $t=1, \ldots, T$,

$$
\begin{aligned}
V_{t}(x, i, j)= & \max _{q_{1} \geq 0, q_{2} \geq 0}\left\{R_{t}(d)-c_{1} \gamma^{1, i} q_{1}-\alpha c_{2} \gamma^{2, j} q_{2}\right. \\
& \underline{d}_{t} \leq d \leq \bar{d}_{t} \\
& -\gamma^{1, i} G_{t}\left(x+q_{1}-d\right)-\left(1-\gamma^{1, i}\right) G_{t}(x-d) \\
& +\alpha \gamma^{1, i}\left(\gamma^{2, j} \mathbf{E}\left[V_{t+1}\left(x+q_{1}+q_{2}-d-\epsilon_{t}, 1,1\right)\right]\right. \\
& \left.+\left(1-\gamma^{2, j}\right) \mathbf{E}\left[V_{t+1}\left(x+q_{1}-d-\epsilon_{t}, 1,0\right)\right]\right) \\
& +\alpha\left(1-\gamma^{1, i}\right)\left(\gamma^{2, j} \mathbf{E}\left[V_{t+1}\left(x+q_{2}-d-\epsilon_{t}, 0,1\right)\right]\right. \\
& \left.\left.+\left(1-\gamma^{2, j}\right) \mathbf{E}\left[V_{t+1}\left(x-d-\epsilon_{t}, 0,0\right)\right]\right)\right\}
\end{aligned}
$$

and $V_{T+1}(x, i, j) \equiv 0$. Note that on the right hand side of Equation (2), the first term in the brackets is the expected revenue; the second and third terms are the expected ordering costs; the value of the next two terms is the expected holding and backlog costs in period $t$; and the remaining terms are the maximum expected total discounted profit-to-go from 
period $t+1$, corresponding to different realizations of the two suppliers' delivery capabilities in period $t$.

For $1 \leq t \leq T$, let $\left(q_{t, 1}^{*}(x, i, j), q_{t, 2}^{*}(x, i, j), d_{t}^{*}(x, i, j)\right)$ denote the optimal solution of $\left(q_{1}, q_{2}, d\right)$ of the maximization problem (2). That is, given state $(x, i, j)$ at the beginning of period $t, q_{t, k}^{*}(x, i, j)$ is the optimal order quantity from supplier $k$ and $d_{t}^{*}(x, i, j)$ is the optimal mean demand. Then, $x+q_{t, 1}^{*}(x, i, j)$ and $x+q_{t, 1}^{*}(x, i, j)-d_{t}^{*}(x, i, j)$ are the optimal target inventory level and the optimal target safety stock level, respectively, when only supplier 1 is considered; similarly, $x+q_{t, 2}^{*}(x, i, j)$ and $x+q_{t, 2}^{*}(x, i, j)-d_{t}^{*}(x, i, j)$ are the optimal target inventory position and the optimal target safety stock position, respectively, when only supplier 2 is considered. Finally, we denote $p_{t}^{*}(x, i, j)=p_{t}\left(d_{t}^{*}(x, i, j)\right)$ as the optimal selling price in period $t$.

In the next section, we will first study the properties of the value function in problem (2), and then characterize the structure of the firm's optimal pricing and inventory policies.

\section{Optimal Policies and Implications}

We begin by examining the properties of the value function in (2). The main technical approach used in this study is lattice and modularity analysis. Some preliminaries on lattice and modularity are provided in the Appendix; and we refer the reader to Topkis (1998) for more discussions. The following proposition establishes important properties of the value function, which will facilitate our analysis on the optimal policies. Below, unless otherwise specified, the proofs will be given in the Appendix.

Proposition 1. For $t=1, \ldots, T, V_{t}(x, i, j)$ is increasing in $i$ and $j$, concave in $x$, and submodular in $(x, i)$ and in $(x, j)$.

Proposition 1 shows that the optimal expected profit-to-go is larger if either one of the suppliers was reliable in the previous period than if it was disrupted in the previous period. In addition, the submodularity results show that, the starting inventory level in each period has a lower marginal value if either one of the suppliers was reliable in the previous period than if it was disrupted. Finally, the concavity of $V_{t}(x, i, j)$ in $x$ implies that the marginal value of inventory decreases if the starting inventory level increases.

With the above properties of the value function, we can derive the structure of the firm's optimal ordering and pricing policies. The following theorem provides a detailed characterization of the firm's optimal policies in each period.
THEOREM 1. For $t=1, \ldots, T$, suppose $(x, i, j)$ is the state of the system at the beginning of period $t$. Then, the firm's optimal policy is determined by critical numbers $\xi_{t, k}(i, j), k=1,2$, such that the firm orders a positive quantity from supplier $k$ if and only if $x<\xi_{t, k}(i, j)$; and both the optimal order quantity $q_{t, k}^{*}(x, i, j)$ and optimal selling price $p_{t}^{*}(x, i, j)$ are decreasing in the starting inventory level $x, k=1,2$. In addition, the optimal policy parameters satisfy the following properties:
(a) $d_{t}^{*}(x, i, j), \quad x-d_{t}^{*}(x, i, j)$, and $x+q_{t, k}^{*}(x, i, j)$ $-d_{t}^{*}(x, i, j)$ are increasing in $x$;
(b) $p_{t}^{*}(x, 1, j) \leq p_{t}^{*}(x, 0, j)$, and $p_{t}^{*}(x, i, 1) \leq p_{t}^{*}(x, i, 0)$;
(c) $q_{t, 1}^{*}(x, 1, j) \geq q_{t, 1}^{*}(x, 0, j)$ and $q_{t, 2}^{*}(x, 1, j)-d_{t}^{*}(x, 1, j)$ $\leq q_{t, 2}^{*}(x, 0, j)-d_{t}^{*}(x, 0, j) ;$ and
(d) $q_{t, 2}^{*}(x, i, 1) \geq q_{t, 2}^{*}(x, i, 0)$, and $q_{t, 1}^{*}(x, i, 1)-d_{t}^{*}(x, i, 1)$ $\leq q_{t, 1}^{*}(x, i, 0)-d_{t}^{*}(x, i, 0)$.

Theorem 1 states that the optimal ordering decision from each supplier in a period is determined by a reorder point (dependent on both suppliers' delivery capabilities in the previous period), above which the firm orders nothing while below which the firm orders a positive amount. In addition, part (a) shows that, when only one supplier is considered, the optimal target safety stock level/position for a period is increasing in the starting inventory level in that period. Part (b) indicates that the optimal selling price in a period is higher when either one of the suppliers was disrupted in the previous period than when it was reliable. Finally, parts (c) and (d) show that the firm would order less from a supplier and keep a higher target safety stock level/position from the other supplier in one period when this supplier was disrupted in the previous period than when it was not. The result in the first part of (c) is intuitive, and (d) is symmetric to (c), thus we only provide an explanation for the second part of (c). When one supplier was disrupted in period $t-1$, to cope with this situation, besides increasing the selling price/lowering customer demand, the firm can also choose to order more from the other supplier. Since ordering more from the other supplier and lowering customer demand are two competing forces to deal with disruption, under our additive demand model it is optimal for the firm to keep a higher level of the optimal order quantity from the other supplier net of the mean demand. Note that if the price is exogenously given in period $t$, then the firm should always order more from the other supplier when one supplier was disrupted in period $t-1$, since it is the only way to deal with disruption. However, this result may not be true if the price is an endogenous decision, since raising the selling price alone can be sufficient to cope with disruption in this case. 
We next study the impacts of unit ordering costs on the firm's optimal profit and the optimal policies, which will reveal some important insights on the impact of dual sourcing. To highlight the dependence on $c_{k}$, we rewrite $V_{t}(x, i, j)$ as $V_{t}\left(x, i, j \mid c_{k}\right)$. Similar notation will be used for the optimal policies and control parameters, whenever needed. Our main results are summarized in the following theorem.

THEOREM 2. For $t=1, \ldots, T$ and $k=1,2$, we have,

(a) $V_{t}\left(x, i, j \mid c_{k}\right)$ is decreasing in $c_{k}$ and supermodular in $\left(x, c_{k}\right)$, while $V_{t}\left(x, i, j \mid c_{k}\right)-c_{k} x$ is submodular in $\left(x, c_{k}\right)$; and

(b) $q_{t, k}^{*}\left(x, i, j \mid c_{k}\right), d_{t}^{*}\left(x, i, j \mid c_{k}\right)$, and $\xi_{t, k}\left(i, j \mid c_{k}\right)$ are decreasing in $c_{k}$; while $p_{t}^{*}\left(x, i, j \mid c_{k}\right)$ and $q_{t, k^{\prime}}^{*}\left(x, i, j \mid c_{k}\right)-d_{t}^{*}\left(x, i, j \mid c_{k}\right)$ are increasing in $c_{k}$ where $k^{\prime}=3-k$.

The monotonicity of the value function $V_{t}$ in $c_{k}$ is rather obvious. The supermodularity and submodularity results in (a) imply that the marginal value of inventory is increasing in $c_{k}$ but by a magnitude less than the increment of $c_{k}$. Theorem 2(b) shows that when the unit ordering cost from one supplier increases, the firm will order less from this supplier, and keep a higher target safety stock level/position for the other supplier; and in the meanwhile, it will charge a higher selling price and hence set a lower mean demand. As a special case, if the selling price is not a decision in period $t$, then the firm will always order more from the other supplier if one supplier increases its unit ordering cost. This result, however, is not always true when the selling price is a decision. The intuitions here are very similar to the ones given above for the case when one supplier was disrupted. In the next section, we will provide a numerical example to demonstrate this point.

An important issue we want to study is the impacts of dual sourcing, that is, for a firm which originally has only one supplier, what are the impacts of introducing a second supplier? Previous research in this area has focused on the impact of dual sourcing on the firm's profit, and here we further explore its impacts on the firm's operational policies, on its customers, and on its suppliers. This can be achieved by applying Theorem 2, since as $c_{k}$ becomes large enough, supplier $k$ will never be used hence the dual-sourcing system would be reduced to a system with a single supplier $k^{\prime}$, where $k^{\prime}=3-k$. Therefore, Theorem 2 enables us to compare a single-sourcing system with a dualsourcing system, and identify the impacts of introducing a second supplier. Specifically, applying Theorem 2 we conclude that introducing a second supplier leads to a higher expected profit and a lower selling price; hence introducing another supplier benefits not only the firm but also its customers. For the incumbent supplier in the single-sourcing system, if the selling price is exogenously given, then it will always receive less orders after the firm introduces the second supplier. However, this result is not always true when the price is an endogenous decision. We will discuss more on this issue in the next section.

Finally, we are interested in understanding the impacts of the reliability of the suppliers. It is straightforward to show, by mathematical induction, that as either one of the two suppliers becomes more reliable (i.e., transition probabilities $\gamma^{k, l}, k=1,2, l=0,1$, increase), the firm's optimal profit function goes up; hence a more reliable supplier is always desired by the firm. However, the impacts of supply reliability on the operational policies are much more complicated. Thus, we mainly study this issue in the next section, where a special case of our general model is considered.

\section{One Reliable and One Unreliable Supplier}

In this section, we focus on the special case where supplier 1 is perfectly reliable and can always deliver the firm's order within the period the order is placed, that is, $\gamma^{1, l}=1$ for $l=1,2$; and only supplier 2 may suffer random disruption, but it is less costly, that is, $c_{1}>c_{2}>0$. (Note that otherwise, supplier 2 will never be utilized.) This could be the case if supplier 1 represents in-house production or a local/domestic reliable supplier, while supplier 2 represents a non-local/ outsourcing supplier. Obviously, all conclusions obtained in the previous section remain valid. We will show below that for this special case the optimal policy has a simpler structure, and more importantly, additional insights can be drawn.

Since supplier 2 is the only supplier which may suffer random disruption, for simplicity we write $J_{t, 2}$ and $\gamma^{2, j}$, respectively, as $J_{t}$ and $\gamma^{j}$ in this section. Besides, the state of the system is now reduced to $(x, j)$, where $x$ is the starting inventory level after receiving delivery from supplier 2 (if any), and $j$ is the delivery capability of supplier 2 in the previous period. Accordingly, the value function $V_{t}(x, i, j)$ can be simplified as $V_{t}(x, j)$, and the optimality equation (2) can be rewritten as, for $t=1, \ldots, T$,

$$
\begin{aligned}
V_{t}(x, j)= & \max _{y_{2} \geq y_{1} \geq x}\left\{R_{t}(d)-c_{1}\left(y_{1}-x\right)-G_{t}\left(y_{1}-d\right)\right. \\
& \underline{d}_{t} \leq d \leq \bar{d}_{t} \\
& +\left(1-\gamma^{j}\right) \alpha \mathbf{E}\left[V_{t+1}\left(y_{1}-d-\epsilon_{t}, 0\right)\right]+\gamma^{j} \\
& \left.\left(-\alpha c_{2}\left(y_{2}-y_{1}\right)+\alpha \mathbf{E}\left[V_{t+1}\left(y_{2}-d-\epsilon_{t}, 1\right)\right]\right)\right\},
\end{aligned}
$$

and $V_{T+1}(x, j)=0$. Here $y_{1}=x+q_{1}$ is the inventory level after ordering from supplier 1, while 
$y_{2}=y_{1}+q_{2}$ is the target inventory position after ordering from both suppliers.

For $1 \leq t \leq T$, let $\left(y_{t, 1}^{*}(x, j), y_{t, 2}^{*}(x, j), d_{t}^{*}(x, j)\right)$ denote the optimal solution of $\left(y_{1}, y_{2}, d\right)$ of the maximization problem (3). That is, $y_{t, 1}^{*}(x, j)$ (respectively, $\left.y_{t, 2}^{*}(x, j)\right)$ is the optimal inventory level after ordering from supplier 1 (respectively, target inventory position after ordering from both suppliers), and $d_{t}^{*}(x, j)$ is the optimal mean demand. Then, $y_{t, 1}^{*}(x, j)-d_{t}^{*}(x, j)$ and $y_{t, 2}^{*}(x, j)-d_{t}^{*}(x, j)$ are the optimal safety stock level for supplier 1 and the optimal target safety stock position for supplier 2, respectively. Note that in the previous section $y_{t, 1}^{*}(x, j)-d_{t}^{*}(x, j)$ is referred to as the optimal target safety stock level for supplier 1 , since in there, the order quantity $q_{t, 1}^{*}(x, j)$ may not be delivered, hence the safety stock level may or may not be reached. In addition, denote $q_{t, k}^{*}(x, j)$ and $p_{t}^{*}(x, j)$ as the optimal order quantity from supplier $k$ and the optimal selling price, respectively, $k=1,2$.

For convenience we define

$$
\begin{gathered}
f_{t}(z, j)=-\alpha c_{2} z+\alpha \mathbf{E}\left[V_{t+1}\left(z-\epsilon_{t}, j\right)\right] ; \\
g_{t}(z, j)=-\left(c_{1}-\alpha c_{2}\right) z-G_{t}(z) \\
\quad+\max _{\bar{z} \geq z}\left\{\gamma^{j} f_{t}(\bar{z}, 1)+\left(1-\gamma^{j}\right) f_{t}(z, 0)\right\}
\end{gathered}
$$

for $t=1, \ldots, T$. Then, the optimality equation (3) can be further simplified to

$$
V_{t}(x, j)=\max _{\substack{y_{1} \geq x \\ \underline{d}_{t} \leq d \leq \bar{d}_{t}}}\left\{R_{t}(d)+c_{1}(x-d)+g_{t}\left(y_{1}-d, j\right)\right\} .
$$

As a corollary of Proposition 1, $V_{t}(x, j)$ is increasing in $j$, concave in $x$, and submodular in $(x, j)$. Since $G_{t}(\cdot)$ is a convex function, it follows from Equations (4) and (5) that both $f_{t}(z, j)$ and $g_{t}(z, j)$ are increasing in $j$, concave in $z$, and submodular in $(z, j)$. For $t=1, \ldots, T$ and $j=0,1$, denote $z_{t, 1}^{*}(j)$ and $z_{t, 2}^{*}$ as the maximizers of $g_{t}(z, j)$ and $f_{t}(z, 1)$, respectively. That is,

$z_{t, 1}^{*}(j)=\arg \max _{z} g_{t}(z, j), \quad$ and $\quad z_{t, 2}^{*}=\arg \max _{z} f_{t}(z, 1)$.

In case that $f_{t}(z, 1)$ (respectively, $\left.g_{t}(z, j)\right)$ is monotonically decreasing in $z$, we define $z_{t, 2}^{*}=-\infty$ (respectively, $\left.z_{t, 1}^{*}(j)=-\infty\right)$. Since $g_{t}(z, j)$ is submodular in $(z, j)$, we have $z_{t, 1}^{*}(1) \leq z_{t, 1}^{*}(0)$. It will be seen later that $z_{t, 1}^{*}(j)$ is the optimal safety stock level for ordering from supplier 1 while $z_{t, 2}^{*}$ is the optimal target safety stock position for ordering from supplier 2. We further define

$$
\hat{d}_{t}=\arg \max _{\underline{d}_{t} \leq d \leq \bar{d}_{t}}\left\{R_{t}(d)-c_{1} d\right\} .
$$

With the preceding notation, the following theorem provides a detailed characterization of the firm's opti- mal ordering and pricing policies in each period. In particular, the ordering policies from both suppliers are of a base-stock type, and the optimal pricing policy is a list-price policy with markdowns, which are much simpler than the optimal policies for the general model with two unreliable suppliers.

TheOREM 3. For $t=1, \ldots, T$, suppose $(x, j)$ is the state of the system at the beginning of period $t$.

(a) The optimal ordering policy from supplier 1 is a base-stock policy with base-stock level $\hat{d}_{t}+z_{t, 1}^{*}(j)$, that is,

$$
y_{t, 1}^{*}(x, j)=\max \left\{\hat{d}_{t}+z_{t, 1}^{*}(j), x\right\} .
$$

(b) The optimal ordering policy from supplier 2 is of a base-stock type with constant target safety stock position $z_{t, 2}^{*}$, that is,

$$
y_{t, 2}^{*}(x, j)=\max \left\{y_{t, 1}^{*}(x, j), d_{t}^{*}(x, j)+z_{t, 2}^{*}\right\},
$$

where $y_{t, 1}^{*}(x, j)$ is given by (7), and $d_{t}^{*}(x, j)$ and $x-d_{t}^{*}(x, j)$ are both increasing in $x$. Thus, an order from supplier 2 is placed only when the inventory level after ordering from supplier 1 is below the base-stock level $d_{t}^{*}(x, j)+z_{t, 2}^{*}$. In particular, when $z_{t, 1}^{*}(j) \geq z_{t, 2}^{*}$, then $y_{t, 2}^{*}(x, j)=y_{t, 1}^{*}(x, j)$ and it is optimal not to order from supplier 2 .

(c) The optimal pricing policy is a list-price policy with markdowns: if $x \leq \hat{d}_{t}+z_{t, 1}^{*}(j)$, then $d_{t}^{*}(x, j)=\hat{d}_{t}$, and a list price $p_{t}^{*}(x, j)=p_{t}\left(\hat{d}_{t}\right)$ is optimal; if $x>\hat{d}_{t}+z_{t, 1}^{*}(j)$, then $p_{t}^{*}(x, j)$ is a decreasing function of both $x$ and $j$, with

$$
p_{t}^{*}(x, j) \leq p_{t}\left(\hat{d}_{t}\right) .
$$

Therefore, the optimal policies for the system with a perfectly reliable quick supplier are much simpler and possess most of the nice properties of singlesourcing inventory models. From Theorem 3(b), $x-d_{t}^{*}(x, j)$ is increasing in $x$ and it is less than or equal to $y_{t, 2}^{*}(x, j)-d_{t}^{*}(x, j)$. Thus, there exists a critical number, such that it is optimal to order nothing from supplier 2 when the starting inventory level in period $t$ exceeds this critical number. It is interesting to note that when supplier 1 is perfectly reliable, it always receives more orders from the firm when supplier 2 was disrupted in the previous period than when it was not, regardless of whether the selling price is an endogenous decision. This result directly follows from Theorem 3(a) and the aforementioned inequality $z_{t, 1}^{*}(1) \leq z_{t, 1}^{*}(0)$; however, this result is not true when supplier 1 is not perfectly reliable, as we discussed in the previous section.

We next analyze the impacts of unit ordering costs on the optimal policies. In addition to all the results in 
Theorem 2, we have the following results when supplier 1 is perfectly reliable.

Proposition 2. For $t=1, \ldots, T, z_{t, 1}^{*}\left(j \mid c_{1}, c_{2}\right) \quad$ is decreasing in $c_{1}$ and increasing in $c_{2} ; z_{t, 2}^{*}\left(c_{1}, c_{2}\right)$ is increasing in $c_{1}$ and decreasing in $c_{2}$; and $q_{t, 1}^{*}\left(x, j \mid c_{2}\right)$ is increasing in $c_{2}$.

Proposition 2 presents some comparative statics results for the safety stock level for supplier $1, z_{t, 1}^{*}(j)$, and the target safety stock position for supplier 2, $z_{t, 2}^{*}$, with respect to the unit ordering costs. Also, it is seen that the firm will order more from supplier 1 when $c_{2}$ increases. In contrast, when $c_{1}$ increases, Theorem 2 shows that $q_{t, 2}^{*}\left(x, j \mid c_{1}\right)-d_{t}^{*}\left(x, j \mid c_{1}\right)$ will increase but it does not specify the effect on the optimal order quantity from supplier 2. When the selling price in period $t$ is exogenously given, we can infer from the above result that the firm will order more from supplier 2. This result, however, is not true when price is also a decision, as demonstrated in the following numerical example.

EXAmple 1. Suppose $T=3, \alpha=0.97, h=1, b=8$, $d_{t}(p)=100-10 p, \quad \varepsilon_{t} \sim$ Uniform $[-20,20], \quad \underline{p}_{t}=0$, $\bar{p}_{t}=8, c_{2}=3, \gamma^{0}=0.3$, and $\gamma^{1}=0.9$. The optimal order quantity from supplier 2 in period 1 when $j=0$ as a function of $c_{1}$ and $x$ is shown in Figure 1. As we can see from this figure, it is clear that the optimal order quantity from supplier 2 is not monotone in $c_{1}$.

Proposition 2 is a powerful result and it allows us to study the operational effect of sourcing diversification. This is because, by letting $c_{1}$ or $c_{2}$ go to infinity, the dual-sourcing problem is reduced to a singlesourcing problem, and the monotonicity result in Theorem 2 and Proposition 2 would enable us to analyze the impact of introducing a second supplier on the incumbent supplier. Applying these arguments we conclude that supplier 1 always receives less orders after the firm introduces the second supplier, regard-

Figure 1 Optimal Order Quantity from Supplier 2 When $j=0$

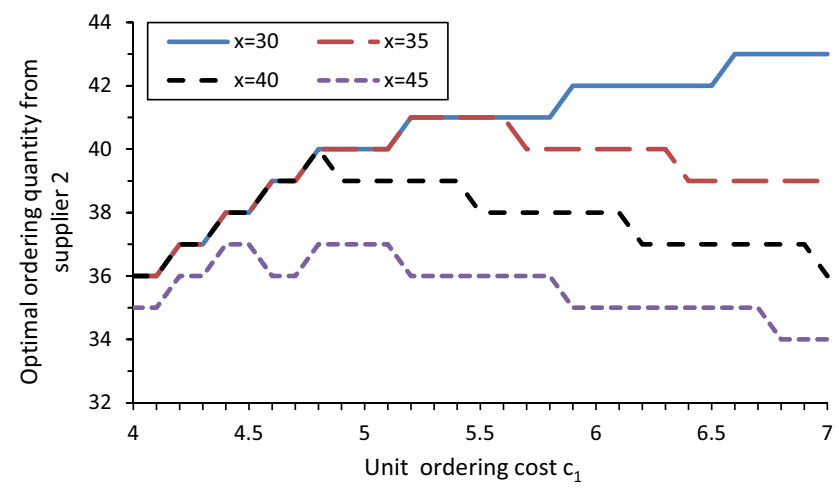

less of whether the selling price is a decision or not; and if the price is exogenously given, supplier 2 also always receives less orders after the firm introduces supplier 1 . However, if the price is a decision, then supplier 2 might receive more orders from the firm after supplier 1 is added into the system. This can be seen from the previous numerical example, say with $x=45$, by comparing the optimal order quantity from supplier 2 for a given $c_{1}$ (say $c_{1}=5$ ) with that for a large enough value of $c_{1}$, which corresponds to another system with only supplier 2 . The intuition behind this is the following: Adding supplier 1 to the system induces two competing forces that may change the optimal order quantity from supplier 2 . On the one hand, it tends to lower the order quantity from supplier 2 due to the newly added supplier 1 ; on the other hand, it will also lower the optimal price thus attract more demand, giving the firm an incentive to order more from supplier 2. When the latter force exceeds the former, the firm will order more from supplier 2.

Remark 2. Following Theorem 2, Proposition 2, and the above discussion, it is interesting to observe that when the selling price of the firm is exogenously given (e.g., the market price), the supplier will receive less orders after the firm introduces a second supplier; but this result is not always true if the selling price is also a decision. Thus, the impact of the firm's pricing decision on its suppliers can be subtle.

We now proceed to study the impacts of supplier 2 's reliability, measured by $\gamma^{0}$ and $\gamma^{1}$ (noting that a larger $\gamma^{l}, l=0,1$, represents a more reliable supplier 2), on the firm's optimal profit functions and operational policies. Since the firm never orders from supplier 2 in period $T$ because of its one-period lead time, neither the optimal profit functions nor the optimal policies in period $T$ depend on $\gamma^{l}$. For $t=1, \ldots, T-1$, we expand the arguments of the optimal profit functions, the optimal policies, and other control parameters to include $\gamma^{l}$, whenever needed, as we did previously on the unit ordering costs. The following theorem summarizes our main results. Among other results, the theorem states that a more reliable supplier is beneficial to both the firm and its customers.

THEOREM 4. For $t=1, \ldots, T-1$ and $l=0,1$, we have

(a) $V_{t}\left(x, j \mid \gamma^{l}\right)$ is increasing in $\gamma^{l}$ and submodular in $\left(x, \gamma^{l}\right)$;

(b) $z_{t, 1}^{*}\left(j \mid \gamma^{l}\right)$ and $z_{t, 2}^{*}\left(\gamma^{l}\right)$ are decreasing in $\gamma^{l}$, and $z_{T-1,2}^{*}\left(\gamma^{l}\right)$ is independent of $\gamma^{l}$; and

(c) $p_{t}^{*}\left(x, j \mid \gamma^{l}\right), q_{t, 1}^{*}\left(x, j \mid \gamma^{l}\right)$, and the optimal target safety stock position $y_{t, 2}^{*}\left(x, j \mid \gamma^{l}\right)-d_{t}^{*}\left(x, j \mid \gamma^{l}\right)$ are 
decreasing in $\gamma^{l}$, while $d_{t}^{*}\left(x, j \mid \gamma^{l}\right)$ and $q_{T-1,2}^{*}$ $\left(x, j \mid \gamma^{l}\right)$ are increasing in $\gamma^{l}$.

Theorem 4(a) shows that the firm's optimal profit function in each period $t$ increases while the marginal value of inventory decreases when supplier 2 becomes more reliable. It is noteworthy that the submodularity of the value function on $\left(x, \gamma^{l}\right)$ plays a key role in analyzing the impact of supplier 2's reliability on the optimal policies. Part (b) shows that the safety stock level for supplier 1 and the target safety stock position for supplier 2 are both decreasing in $\gamma^{l}$, implying that when supplier 2 becomes more reliable, the firm would maintain less safety stocks from both suppliers in managing its inventory. In particular, since supplier 2 is not used in period $T$, it is clear from its definition that $z_{T-1,2}^{*}\left(\gamma^{l}\right)$ does not depend on $\gamma^{l}$.

Part (c) shows that the optimal selling price, the optimal order quantity from supplier 1, and the optimal target safety stock for ordering from supplier 2 are all decreasing in $\gamma^{l}$, which further implies that the optimal mean demand is increasing in $\gamma^{l}$. This is because, when supplier 2 becomes more reliable, the firm is more certain of the delivery of its order hence it will be more economical to order when needed (rather than order more to prepare for future delivery uncertainty).

When the selling price is exogenously given, Theorem 4(c) also implies that the optimal total order quantity in period $t$, which is

$$
q_{t, 1}^{*}\left(x, j \mid \gamma^{l}\right)+q_{t, 2}^{*}\left(x, j \mid \gamma^{l}\right)=y_{t, 2}^{*}\left(x, j \mid \gamma^{l}\right)-x
$$

is decreasing in $\gamma^{l}$. When the selling price is a decision, however, numerical examples can be easily constructed to show that the optimal total order quantity is not monotone in $\gamma^{l}$. Finally, Theorem 4(c) states that the optimal order quantity from supplier 2 is increasing in $\gamma^{l}$ when $t=T-1$. This result, however, again cannot be extended to an arbitrary period $t$, as seen in the following example.

ExAmple 2. Suppose $T=4, c_{1}=5, c_{2}=3, \gamma^{0}=0.3$, and other parameters except $\gamma^{1}$ are the same as those in Example 1. The optimal order quantity from supplier 2 in period 1 when $j=1$ is shown in Figure 2. As can be seen from this figure, the optimal order quantity from supplier 2 can decrease when $\gamma^{1}$ becomes larger.

The underlying intuitions for this phenomenon are as follows. For a period $t$ with $t<T-1$, the firm needs to consider multiple future demands when ordering from supplier 2. When $\gamma^{l}$ is small, the firm probably has to order multiple times until it finally receives an order, which in turn provides the firm

\section{Figure 2 Optimal Order Quantity from Supplier 2 When $j=1$}

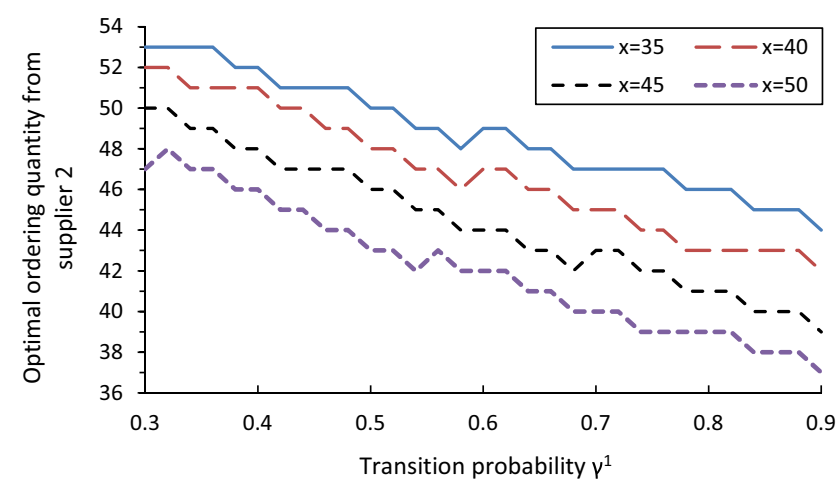

with an incentive to place a large order. In contrast, when $\gamma^{l}$ is large, the firm is quite likely to receive its order after it is placed, thus a small order may be more economical. Therefore, the optimal order quantity from supplier 2 may decrease as $\gamma^{l}$ increases.

REMARK 3. The above discussions present another impact of the firm's pricing decision on the suppliers: when the selling price is exogenously given, the optimal total order quantity decreases when supplier 2 becomes more reliable; however, this result is not always true when the price is an endogenous decision. Note that the impact here is on the optimal total order quantity rather than the optimal order quantity from supplier 2. Thus, regardless of whether the price is a decision or not, supplier 2 may receive less orders when it becomes more reliable.

Having discussed the impact of reliability of the regular supplier (supplier 2), it is natural to ask a similar question about the quick-response supplier (supplier 1). As we pointed out at the end of section 3, although it is straightforward to show that a more reliable supplier is always desired by the firm, the impact of supply reliability on the operational policies is more complicated when both suppliers are unreliable. However, we are able to characterize how the optimal policies change when supplier 1 improves from being unreliable to perfectly reliable. That is, we can compare the general model with both suppliers being unreliable, the one we discussed in the previous sections, with the model studied in this section. The following theorem summarizes our comparison results. We abuse the notations slightly by letting $V_{t}(x, i, j), p_{t}^{*}(x, i, j)$, and $d_{t}^{*}(x, i, j)$ denote our quantities of interest for the model with two unreliable suppliers, while letting $V_{t}(x, j), p_{t}^{*}(x, j)$, and $d_{t}^{*}(x, j)$ denote the corresponding quantities for the model with supplier 1 being perfectly reliable. 
THEOREM 5. For $t=1, \ldots, T$, we have
(a) $V_{t}(x, i, j) \leq V_{t}(x, j)$ and $V_{t}(x, i, j)-V_{t}(x, j)$ is increasing in $x$; and
(b) $p_{t}^{*}(x, i, j) \geq p_{t}^{*}(x, j)$ and $d_{t}^{*}(x, i, j) \leq d_{t}^{*}(x, j)$.

Theorem 5(a) shows that the firm can obtain a higher optimal expected total discounted profit when supplier 1 becomes perfectly reliable from being unreliable, but the incremental benefit is monotonically decreasing in the starting inventory level. That the firm prefers a reliable supplier is obvious; and the result of a diminishing effect of the starting inventory level also appears to be appealing, since the incremental value of sourcing from a perfectly reliable supplier (rather than an unreliable supplier) should become less when the firm has more on-hand inventory. Part (b) states that as supplier 1 becomes perfectly reliable, the firm will charge a lower optimal selling price and hence set a higher optimal mean demand; thus, a reliable supplier also benefits the customers. Hence, these results again support our general claim that, a more reliable supplier benefits both the firm and its customers.

Theorem 5 does not compare the optimal order quantities between the two models. In fact, as demonstrated in the following numerical example, the optimal order quantity from each supplier in one model is not always dominated by that in the other model.

Example 3. Suppose $T=4, c_{1}=5, c_{2}=3$, and the transition probabilities for supplier 2 are $\gamma^{0}=0.3$, and $\gamma^{1}=0.8$ (or, using the notation from Section 2, $\gamma^{2,0}=0.3$, and $\gamma^{2,1}=0.8$ ). In addition, suppose supplier 1 in model 1 is unreliable with $\gamma^{1,0}=0.5$ and $\gamma^{1,1}=0.8$, while it is perfectly reliable in model 2 . Other parameters are the same as those in Example 1. The comparison between the two models of the optimal ordering policies in period 1 when $(i, j)=(1,0)$ is shown in Figure 3. As we can see from this figure, when supplier 1 improves from being unreliable to perfectly reliable, the optimal order quantity from each supplier may be higher or lower, depending on the starting inventory level. Hence, we observe again that a supplier does not always receive more orders from improving its reliability. Another observation from our numerical studies is that the reorder point for ordering from supplier 1 is always higher in model 1 . The intuition is that, when supplier 1 is unreliable, placing multiple rather than a single order can provide diversification over time and reduce the chance of stockout, which implies that it may be optimal to order from supplier 1 in the current period to satisfy future demands in model 1, while it is not optimal to do so in model 2. Thus, the reorder point for ordering from supplier 1 is higher in model 1 .

We conclude this section with a numerical example to demonstrate how dynamic pricing and dual sourcing impact the system performance.

EXAMPLE 4. Suppose supplier 1 is perfectly reliable, $T=5, \quad \gamma^{1}=0.9, \quad c_{1}=5, \quad$ and $(x, j)=(0,0)$. Other parameters are the same as those in Example 1. Figure 4 displays, for different values of $c_{2}$ and $\gamma^{0}$, the percentages of increase in the firm's profit after supplier 2 is introduced to a system which originally has supplier 1 only, assuming the optimal static price (for the given initial state) is used; while Figure 5 shows the percentages of increase in the firm's profit when we further use the optimal dynamic pricing policy in the dual-sourcing system.

From Figures 4 and 5, we can see that supply source diversification can improve the firm's profit very significantly when the selling price is fixed; and the performance can be further improved by using a dynamic pricing policy, but with a less significant magnitude.

Figure 3 Comparison of Optimal Policies When $(i, j)=(1,0)$
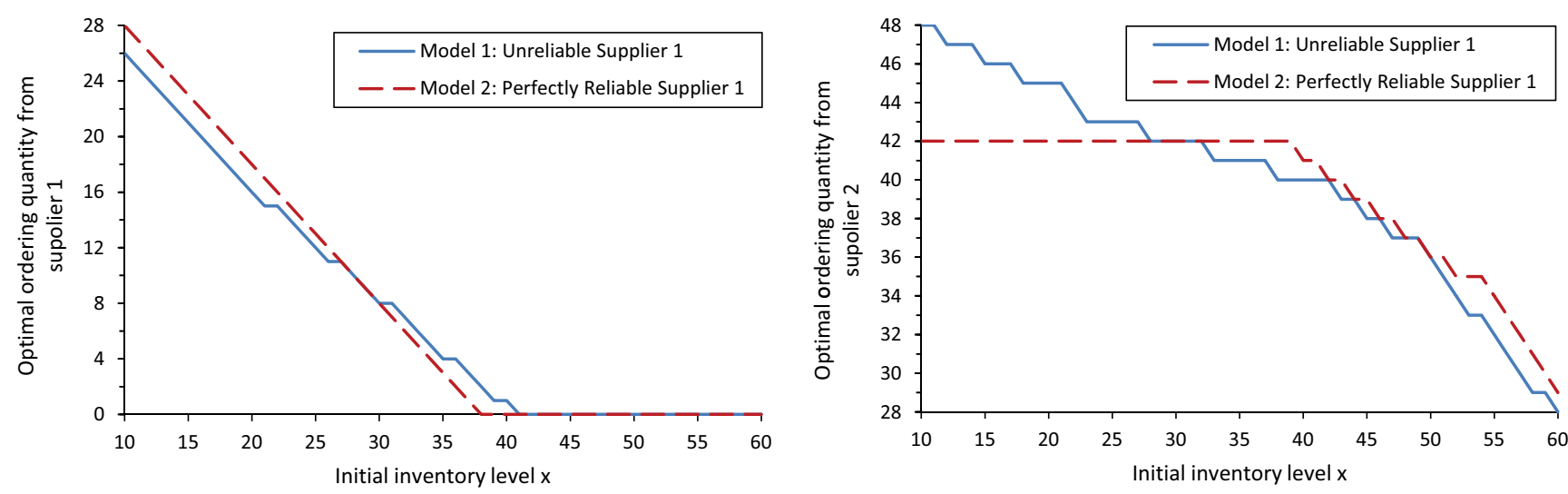
Figure 4 Impact of Dual Sourcing With Static Pricing

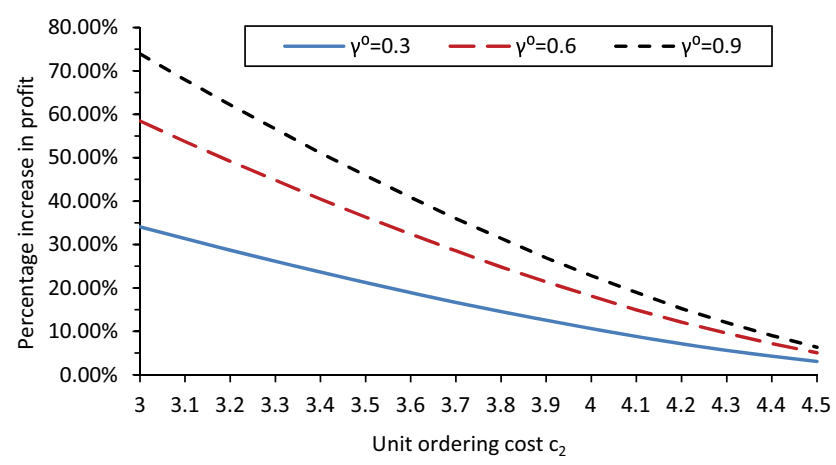

Figure 5 Impact of Dynamic Pricing With Dual Sourcing

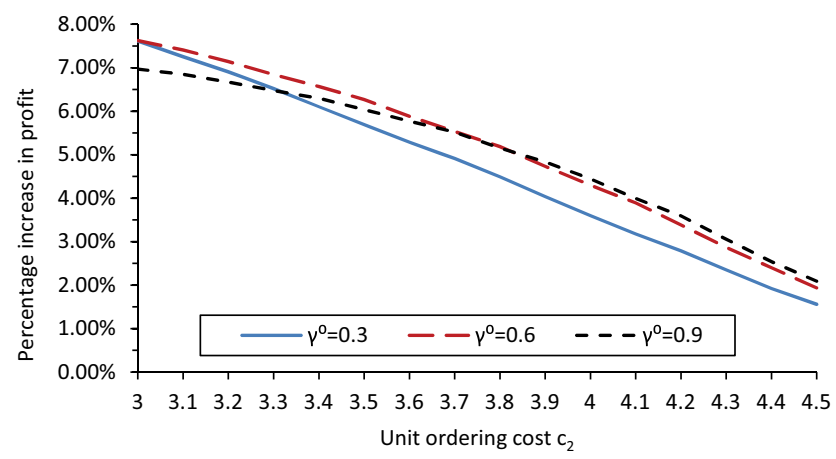

\section{Conclusion and Discussions}

In this study, we study a joint pricing and inventory control problem for a firm that sources from two suppliers, both facing disruption risks. The two suppliers can deliver any order in the same period and in the next period in which the order is placed, respectively, if there is no disruption; but nothing is delivered if disruption occurs. The random disruption processes of the two suppliers are modeled as independent Markov chains. We characterize the firm's optimal pricing and inventory policies over a planning horizon, and identify the impacts of dual sourcing and supply reliability on the firm, on its customers, and on its suppliers. We show that the optimal inventory policy is a reorder point policy, and the optimal price in each period is a decreasing function of the starting inventory level in that period; and for the special case where the quick-response supplier is perfectly reliable, the optimal inventory policy is of a base-stock type and the optimal pricing policy is a list-price policy with markdowns. In addition, we show that both the firm and its customers benefit from dual sourcing and higher supply reliability. For suppliers, we show that if the selling price is exogenously given, then one supplier always receives more orders when the other one was disrupted than when it was not and it always receives less orders after the firm introduces a second supplier; however and interestingly, neither result is true if the price is an endogenous decision. We also find that a supplier does not always receive more orders when it has higher supply reliability.

Our model can be extended along several directions. First, we can show that, if the demands in different periods are independent and identically distributed, and all parameters are stationary over time, then there exist stationary optimal policies for the infinite horizon problem, and all results in this paper remain valid. Second, all of our results except those on the impacts of suppliers' reliability can be extended to the case where the disruption processes of the two suppliers are dependent, as long as the two-dimensional Markov chain describing the supply disruptions are stochastically increasing. Third, we assume in this paper that the firm pays for the delivered product only. This could be easily relaxed by assuming that the firm pays partially for the ordered product and partially for the delivered product, as in Federgruen and Yang (2011). Suppose, in addition to $c_{k}$, the cost for each unit delivered from supplier $k$, $k=1,2$, the firm also pays $c_{k}{ }^{\prime}$ for each unit ordered from supplier $k$. Note that our model is the special case here with $c_{k}^{\prime} \equiv 0$. Then we will need to include a linear term $\left(-c_{1}^{\prime} q_{1}-c_{2}^{\prime} q_{2}\right)$ to the objective function on the right hand side of optimality equation (2). For this case, all of the results in this paper except Theorem 4 can be similarly obtained.

We end this paper with discussions on several other possible extensions of our model.

\subsection{Suppliers with Random Yield}

In this study, the suppliers are assumed to face disruption risks. Given random disruption can be viewed as a special case of random yield with a Bernoulli distribution, it is interesting to consider a similar problem for the case in which the suppliers face general random yield. As mentioned in section 1 , Chen et al. (2013) characterize the structure of the optimal policies for this problem, and show that the optimal inventory policy for each supplier is a near reorder point policy and the optimal price may not be monotone in the starting inventory level. They do not study the impacts of dual sourcing and supply reliability. For the former, it is easy to show that dual sourcing always benefits the firm; but due to the complexity of the problem we are not able to show that it also always benefits the customers. For the latter, if the supplier's reliability is measured according to "convex order" of the random yield, then we can show that the firm always benefits from higher supply reliability; but if it is measured using "stochastic order," then examples can be constructed to show that the firm may not benefit from higher supply reliability. Therefore, we claim that most of our results in this 
paper are for inventory models with multiple sourcing and disruption risks, and not for multiple sourcing with general random yields. (Indeed, it is known that, even for the simplest inventory models, general random yield can lead to very counter-intuitive results, see Gupta and Cooper (2005) on a single-period single-supplier inventory model with random yield).

\subsection{Additive-Multiplicative Demand Models}

In this study, the demand model is assumed to have an additive form. When the demand model takes a more general additive-multiplicative form (i.e., $D_{t}=w_{t} d_{t}(p)+\varepsilon_{t}$, where $w_{t}$ and $\varepsilon_{t}$ are both random variables), following similar analysis to Chen et al. (2013), we can show that the optimal inventory policy is a near reorder point policy for each supplier. In addition, we can prove that the optimal price decreases in the starting inventory level when supplier 1 is perfectly reliable. When supplier 1 is unreliable, we are not able to prove this result theoretically; therefore, we conducted a numerical study; we found that this monotonicity result is true under all the numerical examples we tested. We also conducted several numerical examples to examine the impacts of dual sourcing and supply reliability. In these examples, $w_{t}$ takes discrete distributions with mean $1, \varepsilon_{t}$ is uniformly distributed on $[-a, a]$ for some choices of $a$; and $d(p)$ is linear in $p$. The holding cost $h$ is normalized to 1 ; and several combinations of $c_{1} \in\{5,8\}$, $c_{2} \in\{1,3,6\}$, and $b \in\{8,12\}$ are tested. From these numerical examples, we observe that introducing another supplier and higher supply reliability are both beneficial to the firm and its customers. In addition, if the selling price is a decision, then the incumbent supplier may receive more orders after the firm introduces a second supplier; and the supplier may or may not receive more orders when it becomes more reliable, but it always receives fewer orders when the other supplier becomes more reliable. These results are consistent with our results for the additive demand model. See the online Appendix for an illustration of these numerical results.

\subsection{General Lead Times}

We assume for simplicity that the lead times of the two suppliers are 0 and 1, respectively. It would be more practical to consider the more general model with the lead times being 0 and $k$, respectively. For this general model, the state space needs to be expanded to include pipeline inventories, resulting in a stochastic dynamic program with $k+2$ dimensions. Pang et al. (2012) study a special case of this model with a single perfectly reliable supplier of $k$ periods of lead times, and show that both the optimal order quantity and the optimal price decrease in the onhand and pipeline inventories. For the general model with two suppliers facing disruption risks, by using similar analysis to that in Pang et al. (2012), we can show that (a) the optimal price decreases in the onhand inventory, and (b) the optimal order quantity from each supplier decreases in the on-hand and pipeline inventories when either supplier 1 is perfectly reliable or there is no pricing decision. How ever, due to the complex nature of the problem, we find it very challenging to analyze the impacts of dual sourcing and supply reliability. We expect that new techniques have to be developed to analyze these issues under general settings, thus we leave them for future research.

\section{Acknowledgments}

The authors are grateful to the Senior Editor and three anonymous referees for their constructive comments and suggestions, which have helped us significantly improve the quality and exposition of this paper. The research of Xiting Gong is supported by CUHK Direct Grant under No. 4055022. The research of Xiuli Chao is supported by NSF under CMMI-0927631 and CMMI-1131249. The research of Shaohui Zheng is supported by Hong Kong Research Grants Council under grants \#647611 and \#645212, and by National Natural Science Foundation of China under grant \#71131003.

\section{Appendix}

In this appendix, we provide the proofs of Proposition 1 and Theorems 1,3, and 4. We leave the proofs of the other results in the Appendix S1. Also in the Appendix S1 we will use numerical examples to investigate the additive-multiplicative demand models.

First, some preliminaries are needed. A partially ordered set $X$ is called a lattice if it contains the minimum and maximum of each pairs of its elements; $X^{\prime}$ is called a sublattice of $X$ if it is a subset of $X$ and contains the minimum and maximum (with respect to $X$ ) of each pair of the elements of $X^{\prime}$. Let $V \subset \mathfrak{R}^{n}$ be a lattice and $\mathfrak{R}$ the set of real numbers. A function $f$ : $V \rightarrow \mathfrak{R}$ is called supermodular if

$$
f(\mathbf{x})+f(\mathbf{y}) \leq f(\mathbf{x} \wedge \mathbf{y})+f(\mathbf{x} \vee \mathbf{y})
$$

for any $\mathbf{x}, \mathbf{y} \in V$, where $\wedge$ and $\vee$ are the component-wise minimum and maximum operators, respectively. A function $f$ is submodular if $-f$ is supermodular. For more details on these concepts we refer the reader to Topkis (1998). The following result is from Theorem 2.7.6 and Theorem 2.8.2 in Topkis (1998), which will be frequently used in deriving our theoretical results.

LemMA 1. If $X$ and $Y$ are lattices, $S$ is a sublattice of $X \times Y, f(x, y)$ is supermodular in $(x, y)$ on $S, S_{x}$ is the 
section of $S$ at $x$ in $X$, and $g(x)=\max _{y \in S_{x}} f(x, y)$ is finite, then $g(x)$ is supermodular. In addition, define $y^{*}(x)=\arg \max _{y \in S_{x}} f(x, y)$, then $y^{*}(x)$ is increasing in $x$.

In what follows, we first present two lemmas, which will play important roles in the proofs for Theorems 4 and 5 respectively; and they may be of independent interest; their proofs are given in the Appendix S1.

Lemma 2. Suppose $\phi_{i}(x, \theta): \mathfrak{R} \times[0,1] \rightarrow \mathfrak{R}$ is submodular in $(x, \theta), i=1,2 ; \phi_{1}(x, \theta)$ is concave in $x$; and $\phi_{1}(x, \theta)-\phi_{2}(x, \theta)$ is decreasing in $x$, then

$$
\psi(x, \theta):=\max _{y \geq x}\left\{\theta \phi_{1}(y, \theta)+(1-\theta) \phi_{2}(x, \theta)\right\}
$$

is also submodular in $(x, \theta)$.

Lemma 3. Suppose $\phi_{1}(x, q): \mathfrak{R} \times \mathfrak{R}^{+} \rightarrow \mathfrak{R}$ is concave and submodular in $(x, q)$ and $\phi_{2}(x): \mathfrak{R} \rightarrow \mathfrak{R}$ is concave in $x$. In addition, suppose $\max _{(x, q) \in \Re \times \Re^{+}} \phi_{1}(x, q)=$ $\max _{x \in \Re} \phi_{1}(x, 0)$ and $\phi_{1}(x, 0)-\phi_{2}(x)$ is increasing in $x$. Then,

$$
\psi(x):=\max _{q \geq 0} \phi_{1}(x, q)-\max _{q \geq 0} \phi_{2}(x+q)
$$

is also increasing in $x$.

Proof of Proposition 1. We prove the proposition by induction on $t$. Since $V_{T+1}(x, i, j)=0$, the proposition is obviously true for $t=T+1$. Now we assume inductively that the proposition holds for period $t+1$. In what follows, we shall prove that it also holds for period $t$ and then complete the proof.

We first prove $V_{t}(x, i, j)$ is concave in $x$. Since $R_{t}(\cdot)$ and $-G_{t}(\cdot)$ are both concave functions and $V_{t+1}(x, i, j)$ is concave in $x$ by the inductive assumption, it is easy to verify that the maximand in Equation (2) is concave in $\left(x, q_{1}, q_{2}, d\right)$. Since the constraint in Equation (2) is a convex set, it follows from Proposition B-4 of Heyman and Sobel (1984) that $V_{t}(x, i, j)$ is concave in $x$.

We next prove the remaining results for $V_{t}(x, i, j)$. In what follows, we only prove $V_{t}(x, i, j)$ is increasing in $i$ and submodular in $(x, i)$; and a similar approach can prove that it is increasing in $j$ and submodular in $(x, j)$.

For convenience, we define

$$
\begin{aligned}
L_{t}(x, i, j)= & -\alpha c_{2} x+\alpha \mathbf{E} V_{t+1}\left(x-\epsilon_{t}, i, j\right) ; \\
W_{t}(x, y, i, j)= & -\left(c_{1}-\alpha c_{2}\right) x-G_{t}(x)+\gamma^{2, j} L_{t}(y, i, 1) \\
& +\left(1-\gamma^{2, j}\right) L_{t}(x, i, 0) ;
\end{aligned}
$$

$$
\begin{aligned}
H_{t}(x, y, i, j)= & \max _{q_{1} \geq 0}\left\{\gamma^{1, i} W_{t}\left(x+q_{1}, y+q_{1}, 1, j\right)\right. \\
& \left.+\left(1-\gamma^{1, i}\right) W_{t}(x, y, 0, j)\right\} .
\end{aligned}
$$

Then, the optimality equation (2) can be rewritten as

$$
\begin{aligned}
& V_{t}(x, i, j)= \max _{q_{2} \geq 0}\left\{R_{t}(d)+c_{1}(x-d)+H_{t}(x-d, x\right. \\
& \underline{d}_{t} \leq d \leq \bar{d}_{t} \\
& \\
&\left.\left.+q_{2}-d, i, j\right)\right\} .
\end{aligned}
$$

Since $V_{t+1}(x, 1, j) \geq V_{t+1}(x, 0, j)$, it follows from Equations (A1) and (A2) that

$\max _{q_{1} \geq 0}\left\{W_{t}\left(x+q_{1}, y+q_{1}, 1, j\right)\right\} \geq W_{t}(x, y, 1, j) \geq W_{t}(x, y, 0, j)$.

Since $\gamma^{1,1} \geq \gamma^{1,0}$, it follows from Equation (A3) that $H_{t}(x, y, 1, j) \geq H_{t}(x, y, 0, j)$. Therefore, it follows from Equation (A4) that $V_{t}(x, 1, j) \geq V_{t}(x, 0, j)$.

In what follows, we prove $V_{t}(x, i, j)$ is submodular in $(x, i)$. To this end, we need to first prove that $H_{t}(x, y, i, j)$ is supermodular in $(x, y)$ and submodular in $(x, i)$ and in $(y, i)$.

Since $-G_{t}(x)$ and $V_{t+1}(x, i, j)$ are both concave in $x$, it follows from Equation (A1) that $L_{t}(x, i, j)$ is concave in $x$ and then from Equation (A2) that $W_{t}\left(x-q^{\prime}, y-q^{\prime}, 1, j\right)$ is supermodular in $\left(x, y, q^{\prime}\right)$. Thus, by applying Lemma $1, \max _{q_{1} \geq 0}\left\{W_{t}(x\right.$ $\left.\left.\left.+q_{1}, y+q_{1}, 1, j\right)\right\}=\max _{q^{\prime}} \leq 0 \quad W_{t}(x-q, y-q, 1, j)\right\}$ is supermodular in $(x, y)$. In addition, since $0 \leq \gamma^{1, i}$ $\leq 1$ and $W_{t}(x, y, 0, j)$ is supermodular in $(x, y)$ from (A2), it follows from Equation (A3) that $H_{t}(x, y, i, j)$ is supermodular in $(x, y)$.

Now we prove $H_{t}(x, y, i, j)$ is submodular in $(x, i)$ and in $(y, i)$. Since $V_{t+1}(x, i, j)$ is submodular in $(x, i)$, it directly follows from Equations (A1) and (A2) that $W_{t}(x, y, i, j)$ is submodular in $(x, i)$ and in $(y, i)$. Thus, $W_{t}(x, y, 1, j)-W_{t}(x, y, 0, j)$ is decreasing in $x$ and $y$. For any given $q_{1} \geq 0$, since $L_{t}(x, i, j)$ is concave in $x$ and by Equation (A2), $W_{t}\left(x+q_{1}, y+q_{1}, 1, j\right)-W_{t}(x, y, 1, j)$ is decreasing in $x$ and $y$. Thus, $\max _{q_{1} \geq 0}\left\{W_{t}\left(x+q_{1}, y+\right.\right.$ $\left.\left.q_{1}, 1, j\right)-W_{t}(x, y, 0, j)\right\}$ is also decreasing in $x$ and $y$. From Equation (A3), we have

$$
\begin{aligned}
H_{t}(x, y, 1, j)-H_{t}(x, y, 0, j)=\left(\gamma^{1,1}-\gamma^{1,0}\right) \max _{q_{1} \geq 0} \\
\left\{W_{t}\left(x+q_{1}, y+q_{1}, 1, j\right)-W_{t}(x, y, 0, j)\right\} .
\end{aligned}
$$

Since $\gamma^{1,1} \geq \gamma^{1,0}$, it follows from Equation (A5) that $H_{t}(x, y, 1, j)-H_{t}(x, y, 0, j)$ is decreasing in $x$ and $y$. Thus, $H_{t}(x, y, i, j)$ is submodular in $(x, i)$ and in $(y, i)$. 
We are now ready to prove $V_{t}(x, i, j)$ is submodular in $(x, i)$. By replacing $d$ and $q_{2}$ with $\tilde{d}=x-d$ and $\tilde{q}_{2}=x+q_{2}-d$, Equation (A4) can be rewritten as

$$
V_{t}(x,-i, j)=\max _{\substack{\tilde{q}_{2} \geq \tilde{d} \\ \underline{d}_{t} \leq x-\tilde{d} \leq \bar{d}_{t}}}\left\{R_{t}(x-\tilde{d})+c_{1} \tilde{d}+H_{t}\left(\tilde{d}, \tilde{q}_{2},-i, j\right)\right\} .
$$

Since $H_{t}\left(\tilde{d}, \tilde{q}_{2},-i, j\right)$ is supermodular in $\left(\tilde{d}, \tilde{q}_{2}, i\right)$ and $R_{t}(x-\tilde{d})$ is supermodular in $(x, \tilde{d})$ due to the concavity of $R_{t}(\cdot)$, the maximand in Equation (A6) is a supermodular function in $\left(x, \tilde{d}, \tilde{q}_{2}, i\right)$. In addition, one can easily verify that the constraint in Equation (A6) is a lattice. Thus, by applying Lemma 1, $V_{t}(x,-i, j)$ is supermodular in $(x, i)$ so $V_{t}(x, i, j)$ is submodular in $(x, i)$.

Proof of Theorem 1. We first prove (a). Note that the optimality equation (2) can be written as

$$
\begin{aligned}
V_{t}(x, i, j)= & \max _{\substack{q_{1} \geq 0, q_{2} \geq 0 \\
\underline{d}_{t} \leq d \leq \bar{d}_{t}}}\left\{R_{t}(d)+c_{1}(x-d)\right. \\
& +\gamma^{1, i} W_{t}\left(x+q_{1}-d, x+q_{1}+q_{2}-d, 1, j\right) \\
& \left.+\left(1-\gamma^{1, i}\right) W_{t}\left(x-d, x+q_{2}-d, 0, j\right)\right\} .
\end{aligned}
$$

By replacing $q_{1}, q_{2}$, and $d$ with $\tilde{q}_{1}=-q_{1}$, $\tilde{q}_{2}=x+q_{2}-d$, and $\tilde{d}=x-d$, the optimality equation (A7) can be rewritten as

$$
\begin{aligned}
V_{t}(x, i, j)= & \max _{\tilde{q}_{1} \leq 0, \tilde{q}_{2} \geq \tilde{d}}\left\{R_{t}(x-\tilde{d})\right. \\
& \underline{d}_{t} \leq x-\tilde{d} \leq \bar{d}_{t} \\
& +c_{1} \tilde{d}+\gamma^{1, i} W_{t}\left(\tilde{d}-\tilde{q}_{1}, \tilde{q}_{2}-\tilde{q}_{1}, 1, j\right) \\
& \left.+\left(1-\gamma^{1, i}\right) W_{t}\left(\tilde{d}, \tilde{q}_{2}, 0, j\right)\right\} .
\end{aligned}
$$

Since $R_{t}(\cdot)$ is a concave function, $R_{t}(x-\tilde{d})$ is supermodular in $(x, \tilde{d})$. In addition, from the convexity of $G_{t}(\cdot)$ and the concavity of $V_{t+1}(x, i, j)$ in $x$ from Proposition 1, one can easily verify that $W_{t}\left(d-\tilde{q}_{1}, \tilde{q}_{2}-\tilde{q}_{1}, 1, j\right)$ and $W_{t}\left(\tilde{d}, \tilde{q}_{2}, 0, j\right)$ are both supermodular functions in $\left(\tilde{d}, \tilde{q}_{1}, \tilde{q}_{2}\right)$. Thus, the maximand in Equation (A8) is a supermodular function in $\left(x, \tilde{q}_{1}, \tilde{q}_{2}, \tilde{d}\right)$. Since the constraint in Equation (A8) is a lattice, by applying Lemma $1, \tilde{q}_{1}^{*}(x, i, j)$, $\tilde{q}_{2}^{*}(x, i, j)$ and $\tilde{d}_{t}^{*}(x, i, j)$ are all increasing in $x$. Note that $\tilde{q}_{1}^{*}(x, i, j)=-q_{t, 1}^{*}(x, i, j)$ and $\tilde{q}_{2}^{*}(x, i, j)=x+$ $q_{t, 2}^{*}(x, i, j)-d_{t}^{*}(x, i, j)$, and $d_{t}(x, i, j)=x-d_{t}^{*}(x, i, j)$. Thus, $q_{t, 1}^{*}(x, i, j)$ is decreasing in $x$ while $x-$ $d_{t}^{*}(x, i, j)$ and $x+q_{t, 2}^{*}(x, i, j)-d_{t}^{*}(x, i, j)$ are increasing in $x$. Similarly, it can be shown that $q_{t, 2}^{*}(x, i, j)$ is decreasing in $x$ while $x+q_{t, 1}^{*}(x, i, j)-d_{t}^{*}(x, i, j)$ is increasing in $x$. Now it remains to prove $p_{t}^{*}(x, i, j)$ is decreasing in $x$, or equivalently, $d_{t}^{*}(x, i, j)$ is increasing in $x$. To see this, define

$$
\begin{aligned}
\hat{V}_{t}(z, i, j)= & \max _{q_{1} \geq 0, q_{2} \geq 0}\left\{\gamma^{1, i} W_{t}\left(z+q_{1}, z+q_{1}+q_{2}, 1, j\right)\right. \\
& \left.+\left(1-\gamma^{1, i}\right) W_{t}\left(z, z+q_{2}, 0, j\right)\right\} .
\end{aligned}
$$

Then, the optimality equation (A7) can be simplified and rewritten as

$$
V_{t}(x, i, j)=\max _{\underline{d}_{t} \leq d \leq \bar{d}_{t}}\left\{R_{t}(d)+c_{1}(x-d)+\hat{V}_{t}(x-d, i, j)\right\} .
$$

From the concavity of $V_{t+1}(x, i, j)$ in $x$ from Proposition 1, it easily follows from Equations (A1) and (A2) that $W_{t}(x, y, i, j)$ is jointly concave in $(x, y)$. Then, it follows from Equation (A9) that $\hat{V}_{t}(z, i, j)$ is concave in $z$ and thus $\hat{V}_{t}(x-d, i, j)$ is supermodular in $(x, d)$. Consequently, the maximand in Equation (A10) is supermodular in $(x, d)$. Since the constraint in Equation (A10) is a lattice, by applying Lemma 1, $d_{t}^{*}(x, i, j)$ is increasing in $x$. Part (a) is proved.

For $k=1,2$, define $\xi_{t, k}(i, j)=\sup \left\{x \mid q_{t, k}^{*}(x, i, j)\right.$ $>0\}$. Since $q_{t, k}^{*}(x, i, j)$ is decreasing in $x$ from part (a), $q_{t, k}^{*}(x, i, j)>0$ when $x<\xi_{t, k}(i, j)$ and $q_{t, k}^{*}(x, i, j)=0$ when $x \geq \xi_{t, k}(i, j)$. Thus, a reorder point policy with reorder point value $\xi_{t, k}(i, j)$ is optimal for supplier $k$.

We next prove (b)-(d). We will only prove the results when $j$ is fixed; and a similar approach can prove the results when $i$ is fixed. Note from the proof of Proposition 1 that, the maximand in Equation (A6) is a supermodular function in $\left(x, \tilde{d}, \tilde{q}_{2}, i\right)$ and the constraint in Equation (A6) is a lattice. Then, by applying Lemma $1, \tilde{d}^{*}(x,-i, j)$ and $\tilde{q}_{2}^{*}(x,-i, j)$ are both increasing in $i$. Thus, $\tilde{d}^{*}(x, 1, j) \lesssim \tilde{d}^{*}(x, 0, j)$ and $\tilde{q}_{2}^{*}(x, 1, j) \leq \tilde{q}_{2}^{*}(x, 0, j)$. Note that $\tilde{\tilde{d}}^{*}(x, i, j)=x-d_{t}^{*}(x, i, j)$ and $\tilde{\tilde{q}}_{2}^{*}(x, 1, j)=$ $x+q_{t, 2}^{*}(x, i, j)-d_{t}^{*}(x, i, j)$. Then, $d_{t}^{*}(x, 1, j) \geq$ $d_{t}^{*}(x, 0, j)$ and $q_{t, 2}^{*}(x, 1, j)-d_{t}^{*}(x, 1, j) \leq q_{t, 2}^{*}(x, 0, j)-$ $d_{t}^{*}(x, 0, j)$. Since $p_{t}^{*}(x, i, j)=p_{t}\left(d_{t}^{*}(x, 1, j)\right)$ and $p_{t}(d)$ is decreasing in $d$, we obtain $p_{t}^{*}(x, 1, j) \leq p_{t}^{*}(x, 0, j)$.

Now we prove $q_{t, 1}^{*}(x, 1, j) \geq q_{t, 1}^{*}(x, 0, j)$. Define $\tilde{q}_{1}^{*}(x, y, j)=\arg \max _{\tilde{q}_{1} \leq 0} W_{t}\left(x-\tilde{q}_{1}, y-\tilde{q}_{1}, 1, j\right)$. Then, from the proof of Proposition $1, W_{t}\left(x-\tilde{q}_{1}, y-\tilde{q}_{1}, 1, j\right)$ is a supermodular function in $\left(x, y, \tilde{q}_{1}\right)$. Since the constraint is a lattice, it follows from Lemma 1 that $\tilde{q}_{1}^{*}(x, y, j)$ is increasing in $x$ and $y$. According to Equation (A7), we have the following identity 
$q_{t, 1}^{*}(x, i, j)=-\tilde{q}_{1}^{*}\left(x-d_{t}^{*}(x, i, j), x+q_{t, 2}^{*}(x, i, j)-d_{t}^{*}(x, i, j), j\right)$.

Since $\tilde{q}_{1}^{*}(x, y, j)$ is increasing in $x$ and $y$, $d_{t}^{*}(x, 1, j) \geq d_{t}^{*}(x, 0, j)$ and $q_{t, 2}^{*}(x, 1, j)-d_{t}^{*}(x, 1, j) \leq$ $q_{t, 2}^{*}(x, 0, j)-d_{t}^{*}(x, 0, j)$, we obtain from the above identity that $q_{t, 1}^{*}(x, 1, j) \geq q_{t, 1}^{*}(x, 0, j)$.

Proof of Theorem 3. We first prove (a). Since $g_{t}(z, j)$ is concave in $z$, by the definition of $z_{t, 1}^{*}(j)$, the optimality equation (6) can be rewritten as

$$
\begin{aligned}
V_{t}(x, j)= & \max _{\substack{d_{t} \leq d \leq \bar{d}_{t} \\
d^{\prime}}}\left\{R_{t}(d)-c_{1} d+g_{t}\left(\max \left\{x-d, z_{t, 1}^{*}(j)\right\}, j\right)\right\} \\
& +c_{1} x .
\end{aligned}
$$

Since $g_{t}\left(\max \left\{x-d, z_{t, 1}^{*}(j)\right\}, j\right)$ is increasing in $d$ and equals $g_{t}\left(z_{t, 1}^{*}(j), j\right)$ when $d \geq x-z_{t, 1}^{*}(j)$, it can be easily verified from Equation (A11) that $d_{t_{\hat{A}}}^{*}(x, j)=\hat{d}_{t}$ and $y_{t, 1}^{*}(x, j)=\hat{d}_{t}+z_{t, 1}^{*}(j)$ when $x \leq \hat{d}_{t}+z_{t, 1}^{*}(j)$; while when $x>\hat{d}_{t}+z_{t, 1}^{*}(j)$, we have $\hat{d}_{t} \leq$ $d_{t}^{*}(x, j) \leq x-z_{t, 1}^{*}(j)$ so $y_{t, 1}^{*}(x, j)=x$. Therefore, we obtain $y_{t, 1}^{*}(x, j)=\max \left\{\hat{d}_{t}+z_{t, 1}^{*}(j), x\right\}$.

We next prove (b). By the definition of $f_{t}(z, 1)$ and $z_{t, 2}^{*}$, it can be easily seen from Equation (3) that

$$
\begin{aligned}
y_{t, 2}^{*}(x, j) & =d_{t}^{*}(x, j)+\arg \max _{\bar{y}_{2} \geq y_{t, 1}^{*}(x, j)-d_{t}^{*}(x, j)} f_{t}\left(\bar{y}_{2}, 1\right) \\
& =\max \left\{y_{t, 1}^{*}(x, j), d_{t}^{*}(x, j)+z_{t, 2}^{*}\right\} .
\end{aligned}
$$

In addition, it can be summarized from the first part of the proof that $y_{t, 1}^{*}(x, j) \geq d_{t, 1}^{*}(x, j)+z_{t, 1}^{*}(j)$ for all $(x, j)$. Thus, when $z_{t, 1}^{*}(j) \geq z_{t, 2}^{*}$, we have $y_{t, 1}^{*}(x, j) \geq d_{t}^{*}(x, j)+z_{t, 2}^{*} \quad$ thus $y_{t, 2}^{*}(x, j)=y_{t, 1}^{*}(x, j)$. Finally, the monotonicity results on $d_{t}^{*}(x, j)$ directly follow from Theorem 1.

We finally prove (c). When $x \leq \hat{d}_{t}+z_{t, 1}^{*}(j)$, since $d_{t}^{*}(x, j)=v d_{t}$ from the first part of the proof, it follows that $p_{t}^{*}(x, j)=p_{t}\left(\hat{d}_{t}\right)$. When $x>\hat{d}_{t}+z_{t, 1}^{*}(j)$, the results on $p_{t}^{*}(x, j)$ directly follow from Theorem 1 .

Proof of Theorem 4. We first prove (a) by induction. Since $V_{T+1}\left(x, j \mid \gamma^{l}\right)=0$, (a) is trivially true for $t=T+1$. Now assume inductively that (a) holds for $t+1$. To complete the proof for (a), we shall show that (a) also holds for $t$. By Equation (4) and the inductive assumption on $V_{t+1}\left(x, j \mid \gamma^{l}\right), f_{t}\left(z, j \mid \gamma^{l}\right)$ is submodular in $(z, j)$, increasing in $\gamma^{l}$, and submodular in $\left(z, \gamma^{l}\right)$. From Equation (5),

$$
\begin{aligned}
g_{t}\left(z, j \mid \gamma^{l}\right)= & -\left(c_{1}-\alpha c_{2}\right) z-G_{t}(z)+\max _{\bar{z} \geq z}\left\{\gamma^{2, j} f_{t}\left(\bar{z}, 1 \mid \gamma^{l}\right)\right. \\
& \left.+\left(1-\gamma^{2, j}\right) f_{t}\left(z, 0 \mid \gamma^{l}\right)\right\} .
\end{aligned}
$$

In what follows, we first prove $g_{t}\left(z, j \mid \gamma^{l}\right)$ is increasing in $\gamma^{l}$ and submodular in $\left(z, \gamma^{l}\right)$. Depending on whether $j$ equals $l$, we divide the analysis into two cases.

When $j=l$, it follows from the above properties of $f_{t}\left(z, j \mid \gamma^{l}\right)$ and Lemma 2 that $g_{t}\left(z, j \mid \gamma^{l}\right)$ is submodular in $\left(z, \gamma^{l}\right)$. Now suppose $0 \leq \gamma^{l}<\tilde{\gamma}^{l} \leq 1$. Then,

$$
\begin{aligned}
g_{t}\left(z, l \mid \tilde{\gamma}^{l}\right) & +\left(c_{1}-\alpha c_{2}\right) z+G_{t}(z) \\
& \geq \gamma^{l} \max _{\bar{z} \geq z} f_{t}\left(\bar{z}, 1 \mid \tilde{\gamma}^{l}\right)+\left(1-\gamma^{l}\right) f_{t}\left(z, 0 \mid \tilde{\gamma}^{l}\right) \\
& \geq \gamma^{l} \max _{\bar{z} \geq z} f_{t}\left(\bar{z}, 1 \mid \gamma^{l}\right)+\left(1-\gamma^{l}\right) f_{t}\left(z, 0 \mid \gamma^{l}\right) \\
& =g_{t}\left(z, l \mid \gamma^{l}\right)+\left(c_{1}-\alpha c_{2}\right) z+G_{t}(z),
\end{aligned}
$$

where the first inequality holds since $\max _{\bar{z} \geq z} f_{t}\left(\bar{z}, 1 \mid \tilde{\gamma}^{l}\right) \geq f_{t}\left(z, 1 \mid \tilde{\gamma}^{l}\right) \geq f_{t}\left(z, 0 \mid \tilde{\gamma}^{l}\right)$; and the second inequality holds since $f_{t}\left(z, 0 \mid \gamma^{l}\right)$ and $f_{t}\left(z, 1 \mid \gamma^{l}\right)$ are both increasing in $\gamma^{l}$. Thus, $g_{t}\left(z, l \mid \gamma^{l}\right)$ is increasing in $\gamma^{l}$.

When $j \neq l$, since $f_{t}\left(\bar{z}, 1 \mid \gamma^{l}\right)$ and $f_{t}\left(z, 0 \mid \gamma^{l}\right)$ are both increasing in $\gamma^{l}, g_{t}\left(z, j \mid \gamma^{l}\right)$ is also increasing in $\gamma^{l}$. In addition, Equation (A13) can be rewritten as

$$
\begin{aligned}
g_{t}\left(z, j \mid-\gamma^{l}\right)= & -\left(c_{1}-\alpha c_{2}\right) z-G_{t}(z) \\
& +\max _{\bar{z} \geq z}\left\{\gamma^{2, j} f_{t}\left(\bar{z}, 1 \mid-\gamma^{l}\right)\right. \\
& \left.+\left(1-\gamma^{2, j}\right) f_{t}\left(z, 0 \mid-\gamma^{l}\right)\right\} .
\end{aligned}
$$

Since $f_{t}\left(\bar{z}, 1 \mid-\gamma^{l}\right)$ and $f_{t}\left(z, 0 \mid-\gamma^{l}\right)$ are both supermodular functions and the constraint $\left\{\left(z, \bar{z}, \gamma^{l}\right) \mid \bar{z} \geq z\right\}$ is a lattice, by applying Lemma $1, g_{t}\left(z, j \mid-\gamma^{l}\right)$ is supermodular in $\left(z, \gamma^{l}\right)$ hence $g_{t}\left(z, j \mid \gamma^{l}\right)$ is submodular in $\left(z, \gamma^{l}\right)$.

Now we prove part (a) for period $t$. By replacing the decision variables $y_{1}$ and $d$ as $\tilde{y}_{1}=y_{1}-d$ and $\tilde{d}=x-d$, Equation (6) can be rewritten as

$$
V_{t}\left(x, j \mid-\gamma^{l}\right)=\max _{\substack{\tilde{y}_{1} \geq \tilde{d} \\ d_{t} \leq x-d \tilde{d} \bar{d}_{t}}}\left\{R_{t}(x-\tilde{d})+c_{1} \tilde{d}+g_{t}\left(\tilde{y}, j \mid-\gamma^{l}\right)\right\} .
$$

Since $R_{t}(\cdot)$ is a concave function and $g_{t}\left(\tilde{y}, j \mid-\gamma^{l}\right)$ is supermodular in $\left(\tilde{y}, \gamma^{l}\right)$ and decreasing in $\gamma^{l}$, it can be easily verified from Equation (A14) that $V_{t}\left(x, j \mid-\gamma^{l}\right)$ is decreasing in $\gamma^{l}$ and supermodular in $\left(x, \gamma^{l}\right)$. Thus, $V_{t}\left(x, j \mid \gamma^{l}\right)$ is increasing in $\gamma^{l}$ and submodular in $\left(x, \gamma^{l}\right)$. So (a) is proved.

We next prove (b). Note that $z_{t, 1}^{*}\left(j \mid \gamma^{l}\right)=$ $\arg \max _{z} g_{t}\left(z, j \mid \gamma^{l}\right)$ and $z_{t, 2}^{*}\left(\gamma^{l}\right)=\arg \max _{z} f_{t}\left(z, 1 \mid \gamma^{l}\right)$. Since $g_{t}\left(z, j \mid \gamma^{l}\right)$ and $f_{t}\left(z, 1 \mid \gamma^{i}\right)$ are both submodular in $\left(z, \gamma^{l}\right)$, it directly follows from Lemma 1 that both $z_{t, 1}^{*}\left(j \mid \gamma^{l}\right)$ and $z_{t, 2}^{*}\left(\gamma^{l}\right)$ are decreasing in $\gamma^{l}$. Note that supplier 2 is never used in period $T$ because of its one-period lead time; so $V_{T}(x, j)$ and $f_{T-1}(x, j)$ are 
both independent of $\gamma^{l}$. Hence, by its definition, $z_{T-1,2}^{*}\left(\gamma^{l}\right)$ is independent of $\gamma^{l}$. So (b) is proved.

We finally prove (c). First, from Equation ( A14), it is easily seen that $\tilde{d}^{*}\left(x, j \mid-\gamma^{l}\right)$ is increasing in $\gamma^{l}$. Thus, $\tilde{d}^{*}\left(x, j \mid \gamma^{l}\right)$ is decreasing in $\gamma^{l}$. Since $\tilde{d}^{*}\left(x, j \mid \gamma^{l}\right)=$ $x-d_{t}^{*}\left(x, j \mid \gamma^{l}\right)$ and $p_{t}^{*}\left(x, j \mid \gamma^{l}\right)=p_{t}\left(d_{t}^{*}\left(x, j \mid \gamma^{l}\right)\right)$, it follows that $d_{t}^{*}\left(x, j \mid \gamma^{l}\right)$ is increasing in $\gamma^{l}$ while $p_{t}^{*}\left(x, j \mid \gamma^{l}\right)$ is decreasing in $\gamma^{l}$. Next, from Theorem 3, $y_{t, 1}^{*}\left(x, j \mid \gamma^{l}\right)=\max \left\{\hat{d}_{t}+z_{t, 1}^{*}\left(j \mid \gamma^{l}\right), x\right\}$, and thus, it directly follows from part (b) that $y_{t, 1}^{*}\left(x, j \mid \gamma^{l}\right)$ is decreasing in $\gamma^{l}$. Finally, from Theorem 3, we have

$$
\begin{aligned}
y_{t, 2}^{*}\left(x, j \mid \gamma^{l}\right)-d_{t}^{*}\left(x, j \mid \gamma^{l}\right)= & \max \left\{y_{t, 1}^{*}\left(x, j \mid \gamma^{l}\right)\right. \\
& \left.-d_{t}^{*}\left(x, j \mid \gamma^{l}\right), z_{t, 2}^{*}\left(\gamma^{l}\right)\right\} .
\end{aligned}
$$

Since $y_{t, 1}^{*}\left(x, j \mid \gamma^{l}\right)$ and $z_{t, 2}^{*}\left(\gamma^{l}\right)$ are decreasing in $\gamma^{l}$ while $d_{t}^{*}\left(x, j \mid \gamma^{l}\right)$ is increasing in $\gamma^{l}$, it follows from the above identity that $y_{t, 2}^{*}\left(x, j \mid \gamma^{l}\right)-d_{t}^{*}\left(x, j \mid \gamma^{l}\right)$ is decreasing in $\gamma^{l}$. When $t=T-1$, from Equation (8), we have

$$
\begin{aligned}
q_{T-1,2}^{*}\left(x, j \mid \gamma^{l}\right)= & \max \left\{d_{T-1}^{*}\left(x, j \mid \gamma^{l}\right)-y_{T-1,1}^{*}\left(x, j \mid \gamma^{l}\right)\right. \\
& \left.+z_{T-1,2}^{*}\left(\gamma^{l}\right), 0\right\} .
\end{aligned}
$$

Note that $z_{T-1,2}^{*}\left(\gamma^{l}\right)$ is independent of $\gamma^{l}$ from (b). Thus, $q_{T-1,2}^{*}\left(x, j \mid \gamma^{l}\right)$ is increasing in $\gamma^{l}$. So (c) is proved.

\section{References}

Anupindi, R., R. Akella. 1993. Diversification under supply uncertainty. Manage. Sci. 39(8): 944-963.

Chao, X., H. Chen, S. Zheng. 2008. Joint replenishment and pricing decisions in inventory systems with stochastically dependent supply capacity. Eur. J. Oper. Res. 191(1): 142155.

Chen, X., D. Simchi-Levi. 2004. Coordinating inventory control and pricing strategies with random demand and fixed ordering cost: The finite horizon case. Oper. Res. 52(6): 887896.

Chen, X., D. Simchi-Levi. 2012. Pricing and inventory management. R. Philips, O. Ozer, eds. The Oxford Handbook of Pricing Management. Oxford University Press, Oxford, pp. 785-822.

Chen, J., D. D. Yao, S. Zheng. 2001. Optimal replenishment and rework with multiple unreliable supply sources. Oper. Res. 49(3): 430-443.

Chen, W., Q. Feng, S. Seshadri. 2013. Sourcing from suppliers with random yield for price-dependent demand. Ann. Oper. Res. 208(1): 557-579.

Chopra, S., G. Reinhardt, U. Mohan. 2007. The importance of decoupling recurrent and disruption risks in a supply chain. Nav. Res. Log. 54(5): 544-555.

Dada, M., N. C. Petruzzi, L. B. Schwarz. 2007. A newsvendor's procurement problem when suppliers are unreliable. Manuf. Serv. Oper. Manag. 9(1): 9-32.

Daniel, K. H. 1963. A delivery-lag inventory model with emergency. Chapter 2. H. E. Scarf, D. M. Gilford, M. W. Shelly, eds.
Multistage Inventory Models and Techniques. Stanford University Press, Stanford, California, pp. 32-46.

Federgruen, A., A. Heching. 1999. Combined pricing and inventory control under uncertainty. Oper. Res. 47(3): 454475.

Federgruen, A., N. Yang. 2011. Procurement strategies with unreliable suppliers. Oper. Res. 59(4): 1033-1039.

Feng, Q. 2010. Integrating dynamic pricing and replenishment decisions under supply capacity uncertainty. Manage. Sci. 56(12): 2154-2172.

Feng, Q., R. Shi. 2012. Sourcing from multiple suppliers for price-dependent demands. Prod. Oper. Manag. 21(3): 547563.

Feng, Q., S. P. Sethi, H. Yan, H. Zhang. 2006. Are base-stock policies optimal in inventory problems with multiple delivery modes? Oper. Res. 54(4): 801-807.

Fisher, M., W. Obermeyer, J. Hammond, A. Raman. 1994. Accurate response: the key to profiting from QR. Bobbin 35(February): 48-62.

Fukuda, Y. 1964. Optimal policies for the inventory problem with negotiable leadtime. Manage. Sci. 10(4): 690-708.

Gumus, M., S. Ray, H. Gurnani. 2012. Supply-side story: risks, guarantees, competition, and information asymmetry. Manag. Sci. 58(9): 1694-1714.

Gupta, D. and W. L. Cooper. 2005. Stochastic comparisons in production yield management. Oper. Res. 53(3): 377-384.

Gürler, Ü., M. Parlar. 1997. An inventory problem with two randomly available suppliers. Oper. Res. 45(6): 904-918.

Heyman, D. P., M. J. Sobel. 1984. Stochastic Models in Operations Research, Vol. II. McGraw-Hill, New York.

Li, Q., S. Zheng. 2006. Joint inventory replenishment and pricing control for systems with uncertain yield and demand. Oper. Res. 54(4): 696-705.

Li, T., S. P. Sethi, J. Zhang. 2013a. How does pricing power affect a firm's sourcing decisions from unreliable suppliers? Int. J. Prod. Res. 51(23-24): 6990-7005.

Li, T., S. P. Sethi, J. Zhang. 2013b. Supply diversification with responsive pricing. Prod. Oper. Manag. 22(2): 447-458.

Pang, Z., F. Y. Chen, Y. Feng. 2012. A note on the structure of joint inventory-pricing control with leadtimes. Oper. Res. 60(3): 581-587.

Parlar, M., Y. Wang, Y. Gerchak. 1995. A periodic review inventory model with Markovian supply availability. Int. J. Prod. Econ. 42(2): 131-136.

Shaked, M., J. G. Shanthikumar. 1994. Stochastic Orders and Their Applications. Academic Press, San Diego, CA.

Tomlin, B. 2009. Impact of supply learning when suppliers are unreliable. Manuf. Serv. Oper. Manag. 11(2): 192-209.

Tomlin, B., L. V. Snyder. 2007. On the value of a threat advisory system for managing supply chain disruptions. Working paper. Tuck School of Business. Dartmouth College, Hanover, NH.

Tomlin, B., Y. Wang. 2005. On the value of mix flexibility and dual sourcing in unreliable newsvendor networks. Manuf. Serv. Oper. Manage. 7(1): 37-57.

Topkis, D. M. 1998. Supermodularity and Complementarity. Princeton University Press, Princeton, NJ.

Veeraraghavan, S., A. Scheller-Wolf. 2008. Now or later: a simple policy for effective dual sourcing in capacitated systems. Oper. Res. 56(4): 850-864.

Whittemore, A. S., S. C. Saunders. 1977. Optimal inventory under stochastic demand with two supply options. SIAM J. Appl. Math. 32(2): 293-305. 
Gong, Chao, and Zheng: Dynamic Pricing and Inventory Management Production and Operations Management 23(12), pp. 2058-2074, (C) 2014 Production and Operations Management Society

Yang, Z., G. Aydin, V. Babich, D. R. Beil. 2009. Supply disruptions, asymmetric information, and a backup production option. Manage. Sci. 55(2): 192-209.

Zhao, W., Y. Wang. 2002. Coordination of joint pricing-production decisions in a supply chain. IIE Trans. 34(8): 701-715.

\section{Supporting Information}

Additional Supporting Information may be found in the online version of this article:

Appendix S1: Online Supplement. 